ARTICLE

DOI: $10.1038 / s 41467-017-02633-7 \quad$ OPEN

\title{
A dual role for the N-terminal domain of the IL-3 receptor in cell signalling
}

Sophie E. Broughton ${ }^{1}$, Timothy R. Hercus ${ }^{2}$, Tracy L. Nero' ${ }^{1}$ Winnie L. Kan², Emma F. Barry², Mara Dottore ${ }^{2}$, Karen S. Cheung Tung Shing ${ }^{1,3}$, Craig J. Morton ${ }^{1}$, Urmi Dhagat ${ }^{1}$, Matthew P. Hardy ${ }^{4}$ Nicholas J. Wilson ${ }^{4}$, Matthew T. Downton ${ }^{5}$, Christine Schieber ${ }^{5}$, Timothy P. Hughes ${ }^{6}$, Angel F. Lopez ${ }^{2}$ \& Michael W. Parker (i) ${ }^{1,3}$

The interleukin-3 (IL-3) receptor is a cell-surface heterodimer that links the haemopoietic, vascular and immune systems and is overexpressed in acute and chronic myeloid leukaemia progenitor cells. It belongs to the type I cytokine receptor family in which the $\alpha$-subunits consist of two fibronectin III-like domains that bind cytokine, and a third, evolutionarily unrelated and topologically conserved, N-terminal domain (NTD) with unknown function. Here we show by crystallography that, while the NTD of IL3R $\alpha$ is highly mobile in the presence of IL-3, it becomes surprisingly rigid in the presence of IL-3 K116W. Mutagenesis, biochemical and functional studies show that the NTD of IL3R $\alpha$ regulates IL-3 binding and signalling and reveal an unexpected role in preventing spontaneous receptor dimerisation. Our work identifies a dual role for the NTD in this cytokine receptor family, protecting against inappropriate signalling and dynamically regulating cytokine receptor binding and function.

\footnotetext{
${ }^{1}$ Australian Cancer Research Foundation Rational Drug Discovery Centre, St. Vincent's Institute of Medical Research, Fitzroy, VIC 3065, Australia. ${ }^{2}$ The Centre for Cancer Biology, SA Pathology and the University of South Australia, Adelaide, SA 5000, Australia. ${ }^{3}$ Department of Biochemistry and Molecular Biology, Bio21 Molecular Science and Biotechnology Institute, University of Melbourne, Parkville, VIC 3010, Australia. ${ }^{4}$ CSL Limited, Parkville, VIC 3010, Australia. ${ }^{5}$ IBM Research Australia, Level 5, 204 Lygon Street, Carlton, VIC 3053, Australia. ${ }^{6}$ South Australian Health and Medical Research Institute and University of Adelaide, Adelaide, SA 5000, Australia. Sophie E. Broughton, Timothy R. Hercus and Tracy L. Nero contributed equally to this work. Angel F. Lopez and Michael W. Parker jointly supervised this work. Correspondence and requests for materials should be addressed to A.F.L. (email: angel.lopez@sa.gov.au) or to M.W.P. (email: mparker@svi.edu.au)
} 
nterleukin (IL)-3 is a tightly regulated pleiotropic cytokine produced mainly by activated $\mathrm{T}$ lymphocytes that stimulates the production and function of multiple haematopoietic cell types as well as cells involved in the immune response such as dendritic cells ${ }^{1,2}$. Beyond haemopoiesis and immunity, IL-3 has also been shown to play a role in other biological systems such as angiogenesis ${ }^{3}$ and in the development of the central nervous system $^{4,5}$. In recent years, the IL-3 receptor system has come under the spotlight because stem/progenitor cells from patients with acute myeloid leukaemia (AML) overexpress the IL-3 receptor $\alpha$-subunit (IL3R $\alpha$ ) and this is associated with reduced patient survival ${ }^{3,6-9}$. IL3R $\alpha$ is also overexpressed in chronic myeloid leukaemia (CML) providing a promising target for therapy ${ }^{10}$. Hence, there are ongoing efforts to understand how the IL-3 receptor signals and to develop new therapies in AML and CML based on appropriately targeting IL3R $\alpha$.

IL-3 is a member of the beta common $(\beta c)$ cytokine family, which also includes granulocyte-macrophage colony-stimulating factor (GM-CSF) and IL-5. These cytokines signal through heterodimeric cell-surface receptors that are expressed at low levels and comprise a cytokine-specific $\alpha$-subunit and the shared $\beta$ c subunit ${ }^{1}$. Activation of the IL-3 receptor is thought to involve sequential assembly of a receptor signalling complex whereby the critical step is the initial binding of IL-3 to IL3R $\alpha^{2,11-13}$, followed by recruitment of $\beta c$ and the assembly of a high order complex which, by analogy with the GM-CSF receptor ${ }^{14}$, would bring JAK2 molecules together to trigger downstream signalling. Interestingly, the initial binding of the $\beta c$ cytokines to the $\alpha$-subunits is with low affinity that varies widely among the three receptors, yet the high-affinity binding achieved when $\beta \mathrm{c}$ is present is the same $\left(K_{\mathrm{D}} \sim 200 \mathrm{pM}\right)$ for all three cytokines. IL-3 is remarkable because of its very low affinity for IL3R $\alpha\left(K_{\mathrm{D}} \sim 100-200 \mathrm{nM}\right)$, much lower than the affinity of GM-CSF $\left(K_{\mathrm{D}} 2-10 \mathrm{nM}\right)$ or IL-5 $\left(K_{\mathrm{D}} 1-2 \mathrm{nM}\right)$ for their respective $\alpha$-subunits. However, it is not known how IL3R $\alpha$ recognises IL-3 nor how the IL-3 binary complex overcomes such a low affinity interaction and yet achieves the same high affinity as GM-CSF and IL-5 in the presence of $\beta c$. Previous studies have demonstrated that IL-3 K116 variants, in particular the K116W variant, have higher affinity for IL3R $\alpha$ compared to wild-type IL-3 and enhanced proliferative activity ${ }^{15-17}$. Mutant cytokines with higher affinity for their receptors and/or higher functional potency have been dubbed 'superkines' ${ }^{18}$. A molecular explanation for how a single mutation converts IL-3 into a superkine has not been forthcoming.

We have recently solved the structure of the extracellular region of IL3R $\alpha$ bound to the blocking antibody CSL362 that is currently in clinical trials for $\mathrm{AML}^{11}$. The extracellular region of IL3R $\alpha$ comprises three fibronectin type III (FnIII) domains: an Ig-like N-terminal FnIII domain (NTD) followed by a pair of FnIII domains: domain 2 (D2) and domain 3 (D3). Interestingly, two CSL362:IL3R $\alpha$ complexes were observed, very similar in their overall molecular arrangements, except for the orientation of the IL3R $\alpha$ NTD. The crystals revealed two distinct IL3R $\alpha$ conformations, an 'open' form in which the angle between the NTD and D2 domains of IL3R $\alpha$ is $\sim 95^{\circ}$, and a 'closed' form in which the angle between the NTD and D2 domains is $\sim 55^{\circ}$ and is comparable to the 'wrench-like' conformation ${ }^{11}$ reported for the related cytokine receptors GMR $\alpha^{19}$, IL5R $\alpha^{20,21}, \operatorname{IL} 13 R \alpha 1^{22}$ and IL13R $\alpha 2^{23}$. The reason for these two conformations is unclear but it has been hypothesised that the NTD of IL3R $\alpha$, like other members of the type I cytokine receptor family, may provide molecular flexibility to facilitate cytokine recognition and allow appropriate presentation of cytokine to the signalling subunits of these receptor families. Intriguingly, the NTD is omitted in one isoform of IL3R $\alpha$ (IL3R $\alpha$ SP2) and although not essential, the NTD contributes significantly to wild-type IL-3 function ${ }^{24}$.
Here we present the crystal structures of the IL3R $\alpha$ extracellular domains bound to wild-type IL-3 and to the IL-3 K116W superkine. The structures show the detailed molecular interfaces between IL3R $\alpha$ and ligand and, in particular the mechanism of IL3R $\alpha$ NTD engagement with the cytokine. All-atom explicitsolvent molecular dynamics (MD) simulations reveal that the IL3R $\alpha$ NTD is highly mobile and remains so when bound to wildtype cytokine, but becomes relatively rigid when bound to IL-3 K116W, indicating that the NTD interaction can be distally regulated. Structure-guided mutagenesis, along with functional and biochemical studies, establish a critical role for the IL3R $\alpha$ NTD in optimising IL-3 binding and function and in preventing spontaneous receptor heterodimerisation with $\beta \mathrm{c}$. Given their overall structural conservation, the NTD may perform similar roles in other members of the type I cytokine receptor family.

\section{Results}

IL-3 receptor recognises cytokine differently. We have determined the crystal structure of IL-3 bound to its receptor $\alpha$-subunit, IL3R $\alpha$, to 2.7- $\AA$ resolution (Methods, Table 1, Fig. 1, Supplementary Fig. 1). Two complexes were found in the asymmetric unit and they overlay closely (Supplementary Fig. 2a) with root-mean-square deviation (RMSD) of the C $\alpha$ atoms of $0.7 \AA$ (additional detail in Supplementary Note 1). IL3R $\alpha$ residues P25 to F96 define the Ig-like NTD, residues E108 to F202 define D2 and the third FnIII domain, D3, consists of residues T209 to D294. D2 and D3 constitute the cytokine recognition module (CRM) (Fig. 1a). IL3R $\alpha$ contains $\mathrm{N}$-linked glycans at N80, N109 and N218 (Fig. 1a) that are required for optimal IL3R $\alpha$ expression (Supplementary Fig. 3a, b and see additional detail in Supplementary Note 1).

\begin{tabular}{|c|c|c|}
\hline & $\begin{array}{l}\text { Wild-type IL-3 } \\
\text { binary complex }\end{array}$ & $\begin{array}{l}\text { IL-3 K116W binary } \\
\text { complex }\end{array}$ \\
\hline \multicolumn{3}{|l|}{ Data collection } \\
\hline Space group & $P 6_{5} 22$ & $P 6_{1}$ \\
\hline \multicolumn{3}{|l|}{ Cell dimensions } \\
\hline$a, b, c(\AA)$ & $132.0,132.0,210.6$ & $106.5,106.5,96.1$ \\
\hline$\alpha, \beta, \gamma\left({ }^{\circ}\right)$ & $90,90,120$ & $90,90,120$ \\
\hline Resolution $(\AA)$ & $48.1-2.7(2.83-2.7)$ & $48.1-2.4(2.40-2.39)$ \\
\hline$R_{\text {meas }}$ & $0.19(0.83)$ & $0.08(0.69)$ \\
\hline$R_{\text {pim }}$ & $0.09(0.39)$ & $0.02(0.17)$ \\
\hline $1 / \sigma_{1}$ & $25.7(4.8)$ & $19.7(3.4)$ \\
\hline $\mathrm{CC} 1 / 2$ & $0.99(0.81)$ & $0.99(0.74)$ \\
\hline Completeness (\%) & $100(100)$ & $99.5(97.5)$ \\
\hline Redundancy & $8.1(8.5)$ & $16.1(16.1)$ \\
\hline \multicolumn{3}{|l|}{ Refinement } \\
\hline No. of reflections & 13,304 & 25,439 \\
\hline$R_{\text {work }} / R_{\text {free }}(\%)$ & $22.6 / 28.9$ & $21.6 / 24.2$ \\
\hline \multicolumn{3}{|l|}{ No. of atoms } \\
\hline Protein & 5966 & 5706 \\
\hline Water & 100 & 58 \\
\hline \multicolumn{3}{|l|}{$B$-factors $\left(\AA^{2}\right)$} \\
\hline Main chain & 44.5 & 70.0 \\
\hline Side chain & 45.2 & 72.7 \\
\hline Ligand/ion & 67.6 & 90.0 \\
\hline Water & 32.7 & 59.3 \\
\hline \multicolumn{3}{|l|}{ RMSD } \\
\hline Bond lengths $(\AA)$ & 0.02 & 0.03 \\
\hline Bond angles $\left({ }^{\circ}\right)$ & 2.3 & 2.5 \\
\hline
\end{tabular}

Data were collected from a single crystal per complex. Values in parentheses are for highest resolution shell. Related to Figs. 1, 2, 4, 5, Supplementary Figs. 1, 2 and 4 
a

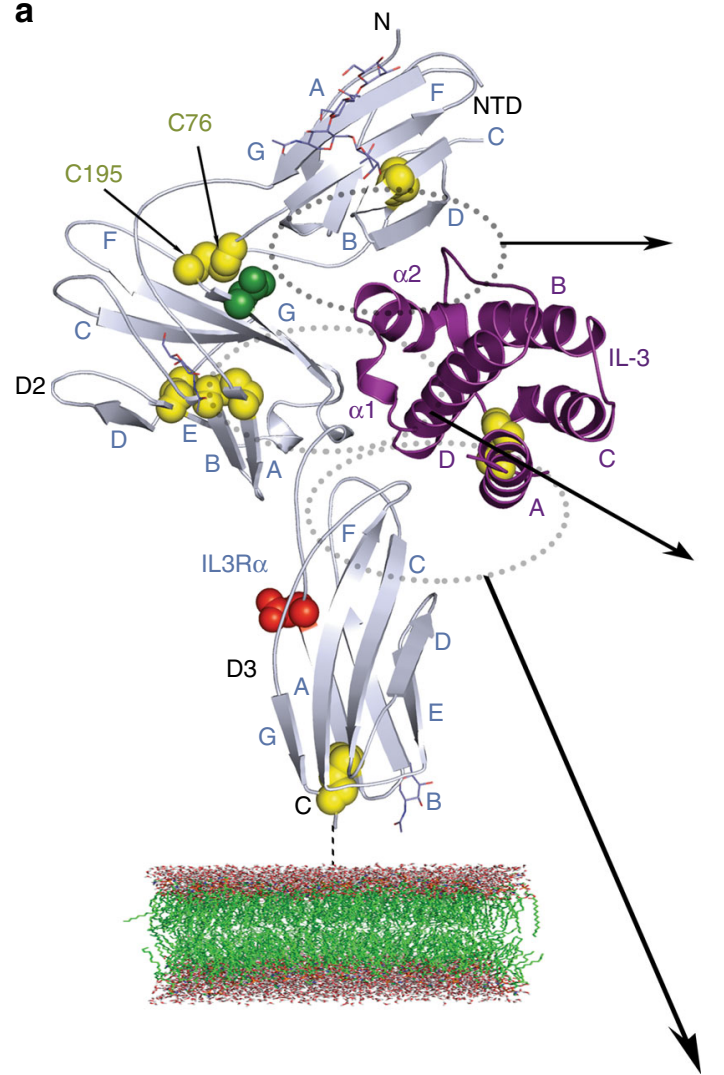

b

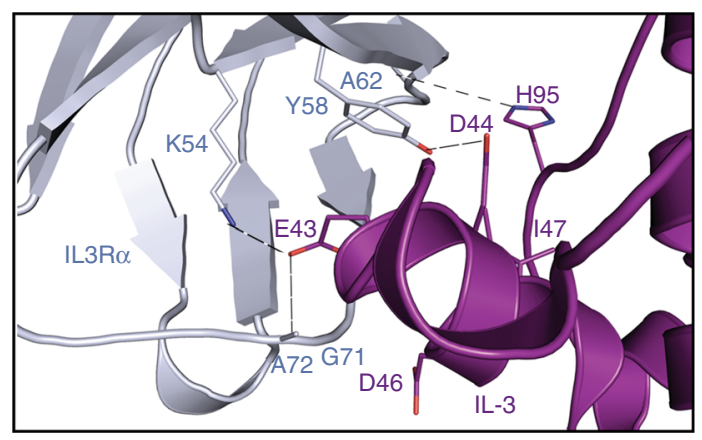

c

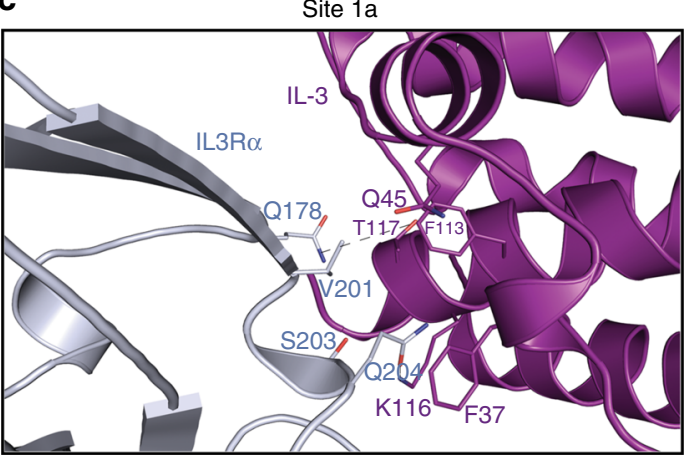

d

Site $1 \mathrm{a}$

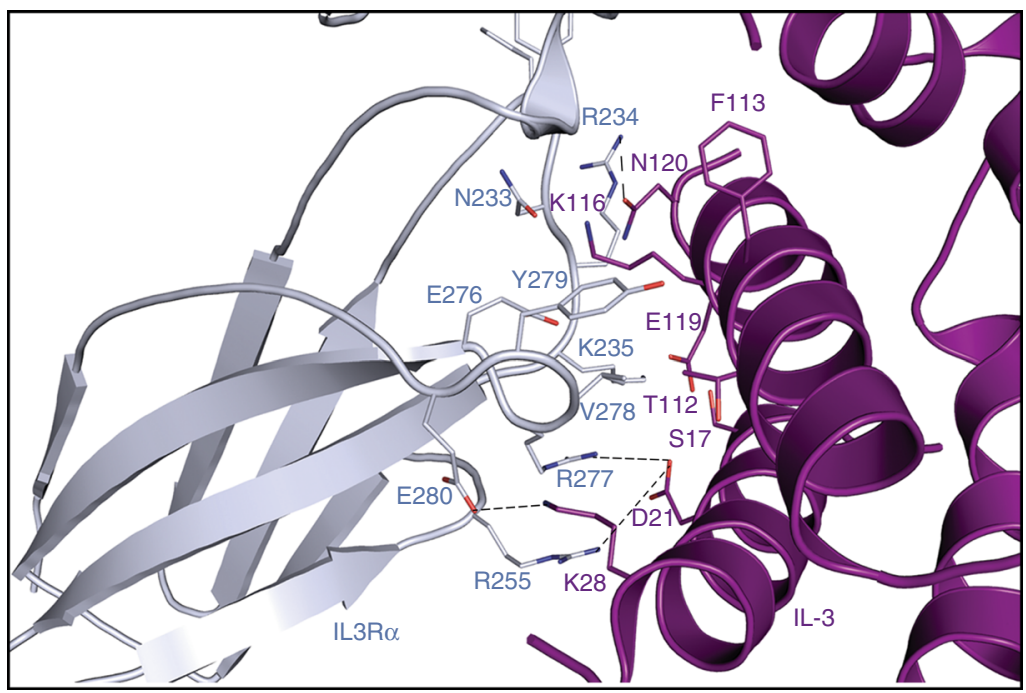

Fig. 1 Crystal structure of the IL-3 binary complex. a The structure of IL3R $\alpha$ (coloured blue-grey in all panels) bound to the four $\alpha$-helical bundle of IL-3 (coloured purple in all panels). The $\beta$-strands that make up the three Fnlll domains (NTD, D2 and D3) and the N-and C-termini are labelled. The cysteine residues in IL3R $\alpha$ are represented as yellow spheres and the bound sugars at N80, N109 and N218 of ILR $\alpha$ are shown as sticks. The mutated N212Q residue is shown as red spheres. D197 is represented as green spheres. b A close-up view highlighting key interactions between IL-3 and the IL3R $\alpha$ NTD (site 1b). Hydrogen bonds are shown as black dashed lines in all panels. c A close-up view highlighting key interactions between IL-3 and the IL3R $\alpha$ D2-D3 linker region (part of site 1a). d A close-up view highlighting key interactions between IL-3 and the IL3R $\alpha$ D3 (part of site 1a). Together, $\mathbf{c}$ and $\mathbf{d}$ define the complete site $1 a$

The three IL3R $\alpha$ FnIII domains adopt a 'wrench-like' conformation that wraps around the four helical bundle of IL3. The hinge region between the D2 and D3 domains has an elbow angle of $\sim 100^{\circ}$, which is within the range observed in related type I cytokine receptor structures ${ }^{11,19-23}$. The elbow angle between D2 and NTD is $\sim 65^{\circ}$ and a disulphide bond (C76-C195) provides an additional covalent interaction between these domains that is not found in related receptors. The receptor component of the IL-3:IL3R $\alpha$ complex is similar to the IL3R $\alpha$ structures previously determined in complex with an antibody $^{11}$; the CRMs are essentially identical (RMSD over the $\mathrm{C} \alpha$ atoms is $0.5 \AA$ ); however, the angle of the NTD with respect to the CRM in the IL-3:IL3R $\alpha$ complex lies between the angles observed in the open and closed forms of the published 
a

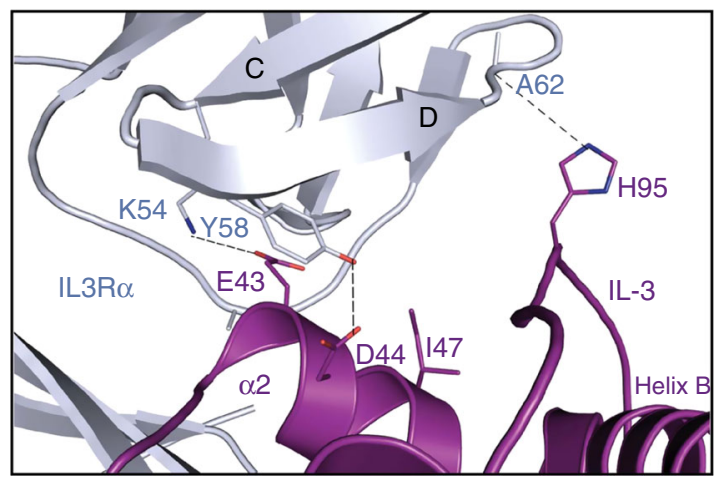

b

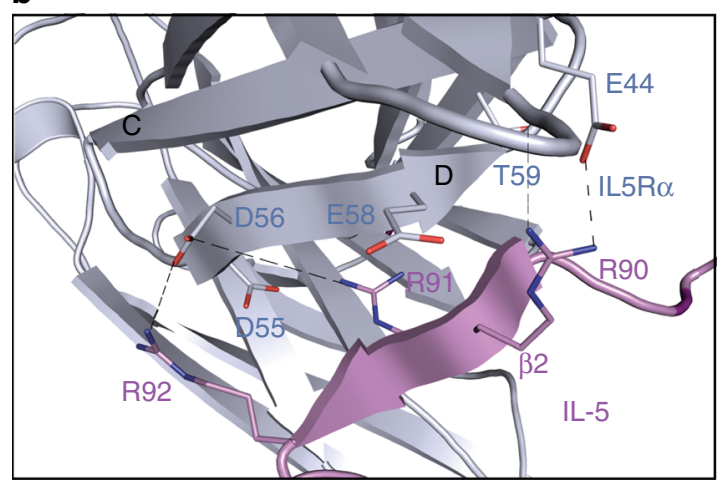

C

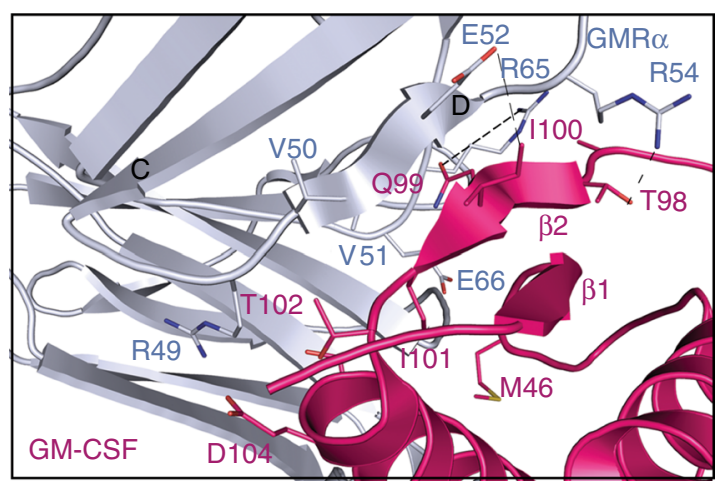

d

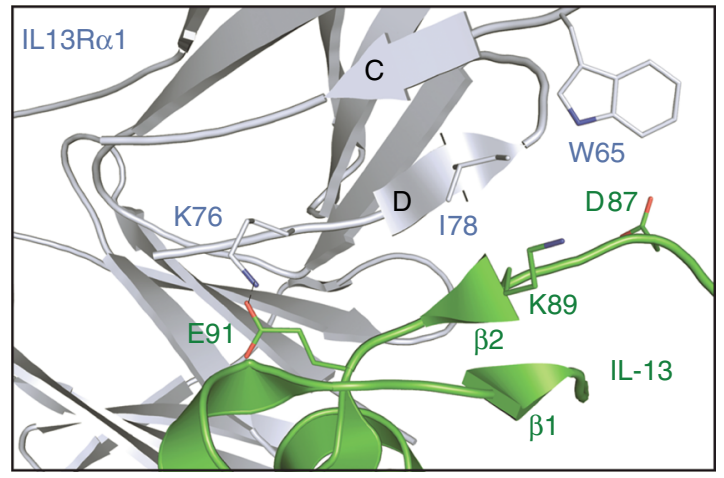

e

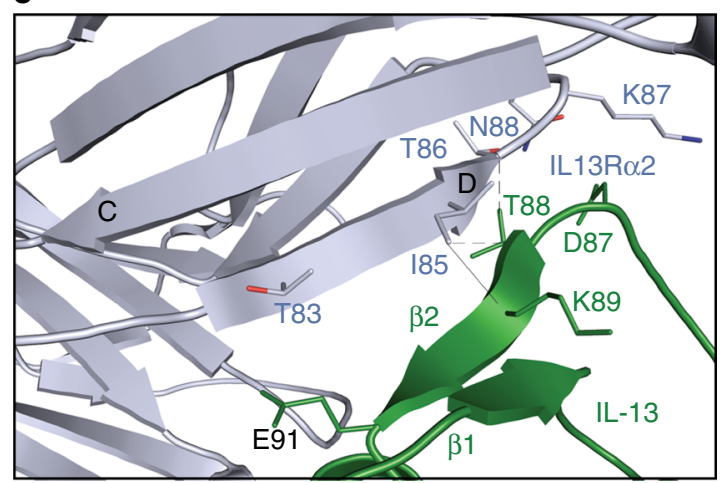

f

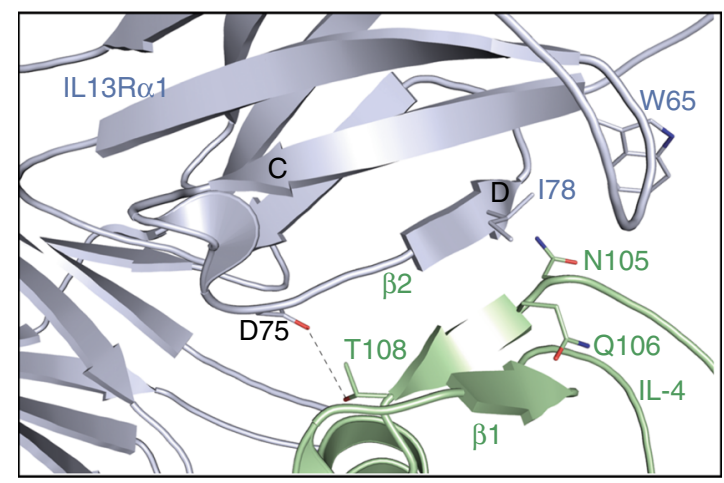

Fig. 2 Comparison of IL3R $\alpha$ site $1 b$ interactions to related cytokine receptor complexes. a Wild-type IL-3:IL3R $\alpha$ binary complex. b IL-5:IL5R $\alpha$ binary complex (PDB ID: 3VA2, 3QT2 ${ }^{20,21}$ ). c GM-CSF:GMR $\alpha$ binary complex (PDB ID: 4RS19). d IL-13:IL13R $\alpha 1$ component of the ternary complex (PDB ID: 3BPO22). e IL13:IL13R $\alpha 2$ binary complex (PDB ID: 3LB6 ${ }^{23}$ ). $\mathbf{f}$ IL-4:IL13R $\alpha 1$ component of the ternary complex (PDB ID: 3BPN ${ }^{22}$ )

structures (Supplementary Fig. $2 \mathrm{~b}$ and additional detail in Supplementary Note 1).

IL3R $\alpha$ contacts IL-3 through an interface containing two distinct interaction sites, site $1 \mathrm{a}$ and site $1 \mathrm{~b}$. The entire interface consists of 12 hydrogen bonds, 4 salt bridges and 16 residues involved in van de Waals interactions; a complete list of these interactions are detailed in Supplementary Table 1. Site 1a comprises the interactions between cytokine and the IL3R $\alpha$ CRM and corresponds to the classical site 1 of simpler type I homodimeric cytokine receptors ${ }^{25}$. The EF loop in D2, the D2-D3 linker region and the BC and FG loops of D3 define the IL3R $\alpha$ contribution to site 1a (Fig. 1c, d, Supplementary Fig. 1c). Site $1 \mathrm{~b}$, the specific molecular interaction between cytokine and the IL3R $\alpha$ NTD, is unique among the $\beta c$ and IL-13 receptors and comprises the C-, D- and E-strands and the DE and EF loops of the IL3R $\alpha$ NTD (Fig. 1b) that interact with IL-3 via the $\alpha 2$-helix, and the CD loop (Fig. 1a, Supplementary Figs. 1c, 4a).
The most surprising difference between the IL-3 binary complex and related cytokine complexes is the manner in which IL-3 interacts at site $1 \mathrm{~b}$. In all related receptor complexes, the cytokine interacts with the receptor subunit NTD via a $\beta$-sheet hydrogen bonding network between the D-strand of the NTD and the $\beta 2$-strand of the cytokine (Fig. 2, Supplementary Fig. 4a and additional detail in Supplementary Note 1). Unlike GM-CSF, IL-4, IL-5 or IL-13, IL-3 does not have a structural equivalent to the $\beta 2$-strand (Fig. 2, Supplementary Fig. $4 \mathrm{a}$ ). In the IL-3 binary complex, the D-strand of the IL3R $\alpha$ NTD is closest to the twoturn IL-3 $\alpha 2$-helix (residues N41-M49, located on the AB loop) and the CD loop. IL-3 makes only five polar interactions with the IL3R $\alpha$ NTD; one between the side chain of IL-3 H95 and the backbone carbonyl of IL3R $\alpha$ A62 and a second between IL-3 D44 (located on the $\alpha 2$-helix) and the hydroxyl group of IL3R $\alpha$ Y58 (Figs. 1b, 2a). In addition, a network of three polar interactions between IL-3 E43 and IL3R $\alpha$ K54, G71 and A72 (Figs. 1b, 2a) 

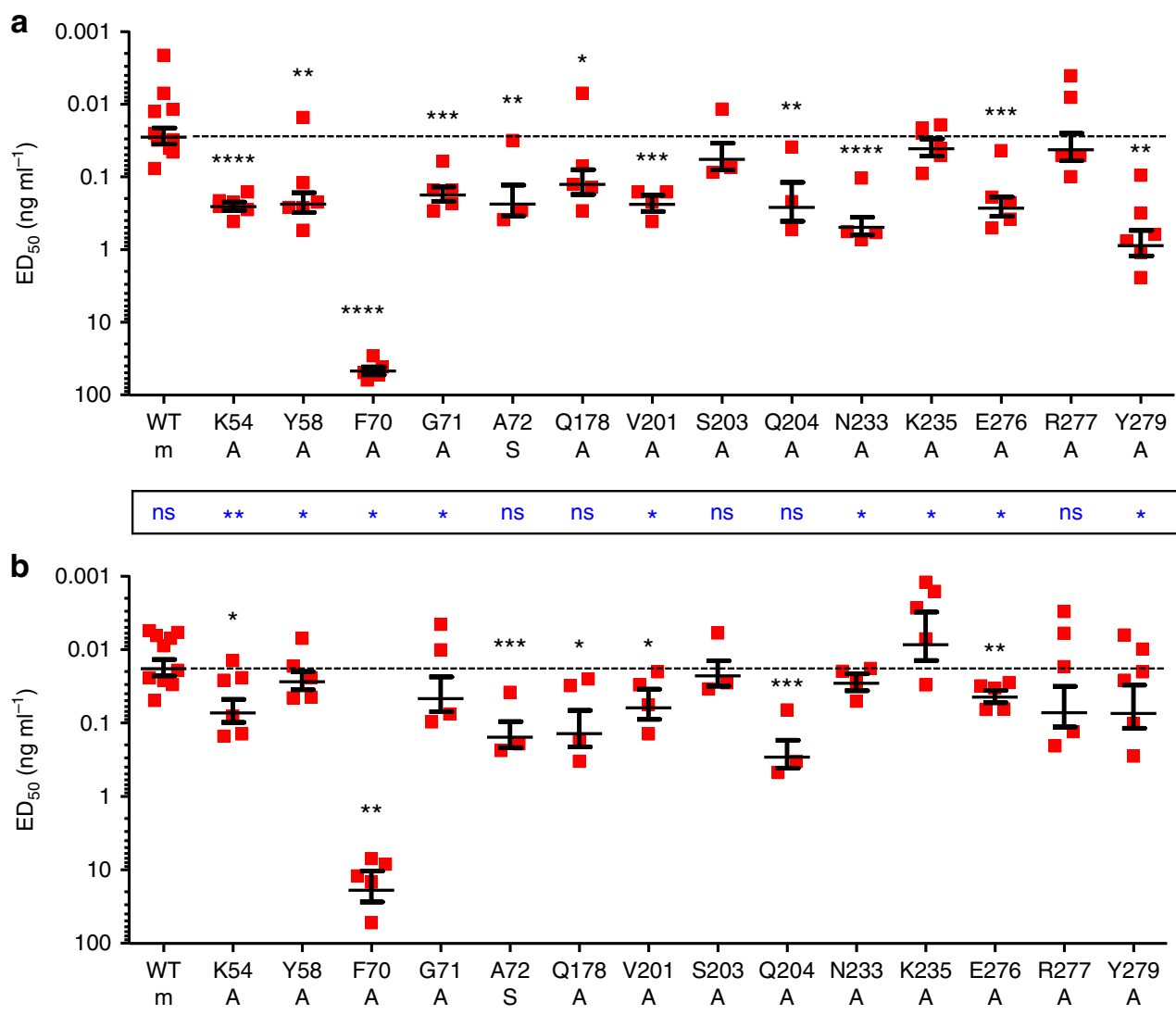

Fig. $\mathbf{3}$ IL3R $\alpha$ site $1 \mathrm{~b}$ residues are a functional hotspot in mediating IL-3 signalling. Functional activity of IL3R $\alpha$ mutants was measured in $\mathbf{a}$ wild-type IL-3 or $\mathbf{b}$ IL-3 K116W dose-response proliferation assays using CTLL-2 cell lines transduced to co-express the $\beta$ c subunit with either wild-type or mutant forms of IL3R $\alpha$. The identity of each IL3R $\alpha$ mutant is shown along with the substituted residue $(\mathrm{m})$. Half-maximal responses (ED 50 ) were calculated from each experiment and averaged. The dashed line represents the response from cells expressing wild-type IL3R $\alpha$. Errors represent SEM. Statistical significance of differences in functional response between wild-type IL3R $\alpha$ and the mutant forms of IL3R $\alpha(p)$ was determined using a two-tailed unpaired $t$ test and are shown as black asterisks above the data for each mutant. Statistical significance of differences in functional response between wild-type IL-3 and IL-3 $\mathrm{K} 116 \mathrm{~W}(p)$ was also determined using a two-tailed unpaired $t$ test and are shown as blue asterisks between data sets $\mathbf{a}$ and $\mathbf{b}$. ns $p>0.05,{ }^{\star} p<0.05$, ${ }^{\star \star} p<$ $0.01,{ }^{* \star *} p<0.001,{ }^{* \star *} p<0.0001$

further stabilises the IL-3:IL3R $\alpha$ NTD (site $1 b$ ) interaction. The IL-3 $\alpha 2$-helix tucks into a crevice between the D-strand and the EF loop of the IL3R $\alpha$ NTD and it is a unique point of stabilisation that does not occur in GM-CSF, IL-4, IL-5 or IL-13 (Fig. 2, Supplementary Fig. 4a). A detailed analysis of the site 1a and site $1 \mathrm{~b}$ interaction surfaces in the IL-3:IL3R $\alpha$ binary complex structure shows that multiple IL-3 residues contribute to IL3R $\alpha$ binding (Supplementary Table 1).

NTD contributes to optimal IL-3 binding and signalling. We have recently identified functional roles for a distinct set of IL3R $\alpha$ residues in mediating direct IL-3 binding and from our crystal structure we observe that these residues are distributed across both site $1 \mathrm{a}$ and site $1 \mathrm{~b}$ (Fig. 1, Supplementary Table 1) ${ }^{11}$. In site 1a, Q178 in the EF loop of D2, V201 and S203 in the D2/D2-D3 linker region, N233 in the BC loop of D3 and E276, R277 and Y279 in the F-strand/FG loop of D3-all have a functional role. In site $1 \mathrm{~b}$, the key functional residues are K54 in the C-strand, Y58 in the D-strand and G71 and A72 in the EF loop of the NTD.

We previously used surface plasmon resonance and the soluble form of the $\alpha$-subunit (ie, extracellular domain only, $\operatorname{sIL} 3 \operatorname{R} \alpha$ ) to investigate the contribution of these IL3R $\alpha$ residues to IL-3 binding ${ }^{11}$. Compared to wild-type sIL3R $\alpha\left(K_{\mathrm{D}}=220 \mathrm{nM}\right)$, alanine mutations of Y58, G71 in the NTD, and E276 or Y279 in site 1a abolished measurable IL-3 binding $\left(K_{\mathrm{D}}>5000 \mathrm{nM}\right)$, while alanine mutations of site 1a Q178, V201, S203, N233 and R277 produced modest reductions in IL-3-binding affinity $\left(K_{\mathrm{D}}=580-2250 \mathrm{nM}\right)$. These differences in IL-3 binding became masked when fulllength receptor mutants were co-expressed with $\beta c$ on COS cells and wild-type high-affinity binding was restored ${ }^{11}$ while also demonstrating that the loss of measurable IL-3 binding does not neccessarily mean abolition of IL-3 interaction. Despite this, CTLL-2 cells co-expressing $\beta c$ and many of the IL3R $\alpha$ mutants (Supplementary Fig. 5) are significantly less responsive to IL-3 stimulation in cell proliferation studies than cell expressing wildtype IL3R $\alpha$ (Fig. 3, Supplementary Table 2). In particular, mutation of residues K54, Y58, F70, G71 and A72 in the NTD all led to significant reductions in IL-3 function $\left(\mathrm{ED}_{50}=0.18-46.6\right.$ $\left.\mathrm{ng} \mathrm{ml}^{-1}\right)$ compared to wild-type IL3R $\alpha\left(\mathrm{ED}_{50}=0.028 \mathrm{ng} \mathrm{ml}^{-1}\right)$. In the IL3R $\alpha$ CRM region, only mutation of Q178, V201, Q204, N233, E276 and Y279 led to a significant reduction in IL-3 function (Fig. 3, Supplementary Table 2). The reduction in IL-3 signalling we observe for these IL3R $\alpha$ mutations may arise as a result of reduced IL-3 binding to IL3R $\alpha$, but may also reflect subtle alterations in the engagement of $\beta c$ in IL-3 signalling complexes utilising mutant IL3R $\alpha$. Mutation of the buried F70 residue has a drastic effect on IL-3 responsiveness $\left(\mathrm{ED}_{50}=46.6\right.$ $\mathrm{ng} \mathrm{ml} \mathrm{I}^{-1}$ ); however, this may be attributed to a major reduction in the expression of the IL3R $\alpha$ F70A mutant (Supplementary Fig. 5). 
a

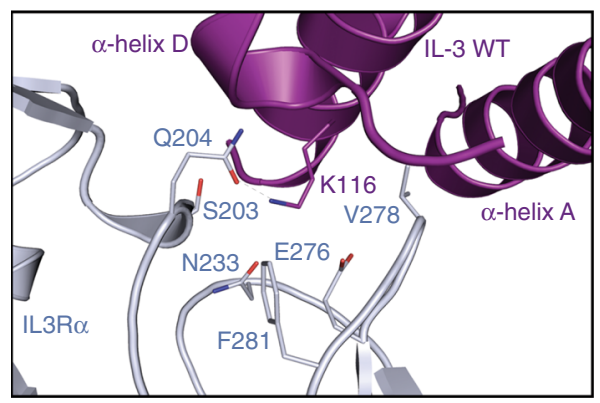

C

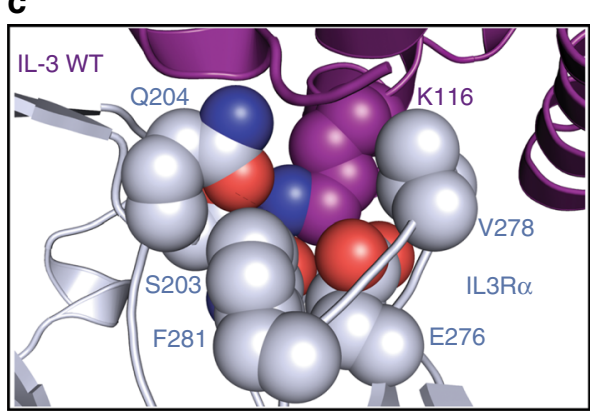

b

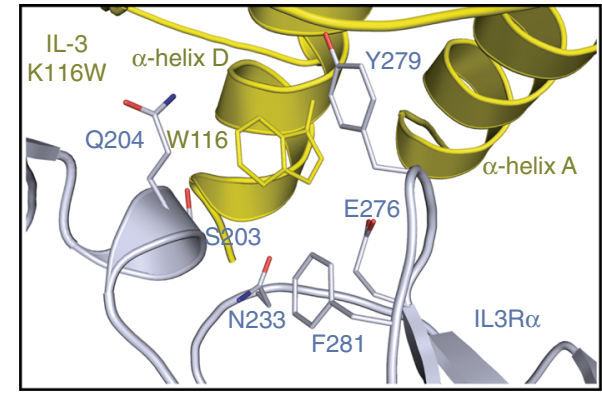

d

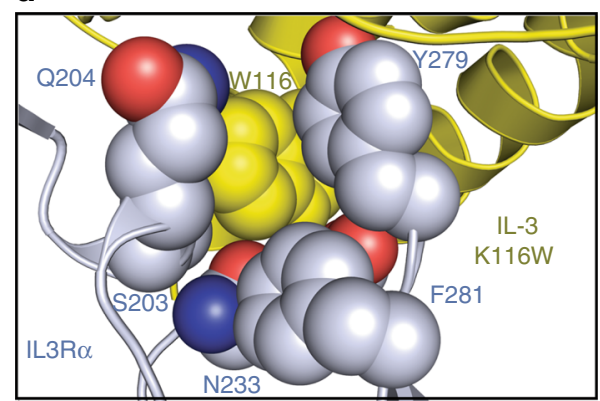

Fig. 4 Structure of IL3R $\alpha$ bound to IL-3 K116W. a Close-up view of the environment around IL-3 K116 in site 1a of the wild-type IL-3 binary complex. Colour scheme as described for Fig. 1b-d. b Close-up view of the environment around W116 in site 1a of the IL-3 K116W binary complex. IL3R $\alpha$ coloured blue-grey and IL-3 K116W coloured yellow. c Residues around IL-3 K116 in the wild-type IL-3 binary complex displayed as spheres to demonstrate the volume they occupy and the residue packing at this site. $\mathbf{d}$ As for c, but for the IL-3 K116W binary complex

These experiments identify multiple residues in IL3R $\alpha$ that are distributed across site $1 \mathrm{a}$ and site $1 \mathrm{~b}$, which contribute significantly to IL-3 function.

IL3R $\alpha$ recognises IL-3 $\mathrm{K} 116 \mathrm{~W}$ via a network of interactions. To further explore the role of site 1 in IL-3 binding and signalling, we determined the crystal structure of IL-3 K116W bound to IL3R $\alpha$ at 2.4- $\AA$ resolution (Methods, Table 1, Supplementary Fig. 1). The IL-3 and IL-3 K116W binary complex structures are essentially identical (Supplementary Fig. 2b) with an overall RMSD over the C $\alpha$ atoms of $0.6 \AA$ (RMSD for IL3R $\alpha$ is $0.5 \AA$ and for IL-3 is $0.3 \AA$ ). The cytokine buried surface area is similar in both complexes (1150 $\AA^{2}$ for IL-3 vs $1166 \AA^{2}$ for IL-3 K116W), with close surface complementarity values $\left(S_{c}=0.69\right.$ for IL-3 vs 0.71 for IL-3 K116W) and a similar number of polar interactions between the cytokine and IL3R $\alpha$ (11 hydrogen bonds (one less than wild type), 5 salt bridges (one more than wild type)).

Not surprisingly, the most significant differences between the wild-type IL-3 and IL-3 K116W structures occur within the vicinity of residue 116 (Fig. 4). Within the cytokine itself, the single-turn $\alpha 1$-helix located on the wild-type IL-3 AB loop unfurls slightly in IL-3 K116W (Supplementary Fig. 4b, d) to allow F37 to interact via edge-to-face $\pi-\pi$ interactions with W116, which in turn interacts with F113 (also via edge-to-face $\pi-\pi$ interactions). The $\pi$ $\pi$ interaction network formed between F37-W116-F113 stabilises the cytokine $\mathrm{AB}$ loop and reduces the loop's overall mobility as assessed by comparisons of the C $\alpha$ RMSD for the cytokines alone (200 ns MD simulation, Supplementary Fig. 4b, c) and C $\alpha$ contact distances between F37, F113 and K/W116 in the binary complexes (100 ns MD simulations, Supplementary Fig. 7b, c) (additional detail in Supplementary Note 1). In the wild-type IL-3 binary complex, the IL-3 K116 side chain points directly into an IL3R $\alpha$ hydrophobic pocket lined by residues Q204, N233, V278, Y279 and F281, and is further capped by F37 in IL-3 (Fig. 4a, c). The IL3 K116 side chain is stabilised by polar interactions with S203,
Q204 and N233 in IL3R $\alpha$, and N120 in IL-3. In contrast, the W116 side chain of IL-3 K116W slots into a groove formed by Q204 and Y279 of IL3R $\alpha$, and forms edge-to-face $\pi-\pi$ interactions with F37 and F113 in IL-3, and Y279 and F281 in IL3R $\alpha$. This results in the IL-3 K116W side chain sitting snuggly in a site 1a pocket formed by F37, T112, F113, T117, E119 and N120 of IL-3 and S203, Q204, N233, E276, V278 and Y279 of IL3R $\alpha$ (Fig. 4b, d). Additional interactions with the IL-3 K116W side chain also have a downstream effect on the adjacent C-terminal end of helix D in IL3 , which is involved in van der Waals contacts with IL3R $\alpha$ via residues E119, A121 and A123.

Differing effects of site 1 residues on IL-3 K116W function. We assessed the impact of IL $3 \mathrm{R} \alpha$ site 1 mutations on IL-3 K116W function using the CTLL- 2 cell lines co-expressing $\beta \mathrm{c}$ and IL3R $\alpha$ mutants (Fig. 3, Supplementary Table 2). In general, mutations of IL3R $\alpha$ site 1 residues had less impact on IL-3 K116W function than for wild-type IL-3. We observed that mutation of residues K54, A72, Q178, V201, Q204 and E276A led to significant reductions in IL-3 K116W function $\left(\mathrm{ED}_{50}=0.044-0.29 \mathrm{ng} \mathrm{ml}^{-1}\right.$ ) compared to wild-type IL3R $\alpha\left(\mathrm{ED}_{50}=0.018 \mathrm{ng} \mathrm{ml}^{-1}\right)$. As for wild-type IL-3, the drastic impact of the IL3R $\alpha$ F70A mutation on IL-3 K116W responsiveness $\left(\mathrm{ED}_{50}=18.8 \mathrm{ng} \mathrm{ml}^{-1}\right)$ can be largely attributed to reduced IL3R $\alpha$ F70A expression levels (Fig. 3, Supplementary Fig. 5, Supplementary Table 2).

IL3R $\alpha$ S203, Q204, N233 and Y279 all interact directly with both K116 of IL-3 and W116 of IL-3 K116W (Fig. 4, Supplementary Table 1). Interestingly, both cytokines display significantly reduced functional activity on CTLL-2 cells expressing the IL3R $\alpha$ Q204A mutant (Fig. 3, Supplementary Table 2). In contrast to Q204, mutation of S203 does not affect the function of either cytokine while mutation of N233 or Y279 significantly reduces the function of wild-type IL-3 only suggesting that these residues directly influence the K116/W116 cytokine interaction. Mutation of IL3R $\alpha$ residues in the vicinity of the IL-3 K116/W116 
Table 2 Enhanced site 1a interactions do not fully compensate for the loss of site $1 \mathrm{~b}$ binding interactions through the NTD

\begin{tabular}{|c|c|c|c|c|c|c|c|c|}
\hline Cell & Receptor subunits & \multicolumn{3}{|c|}{ Wild-type IL-3 } & \multicolumn{3}{|c|}{ IL-3 K116W } & $\boldsymbol{P}$ \\
\hline CTLL-2 & $\mathrm{IL} 3 \mathrm{R} \alpha+\beta \mathrm{c}$ & 2 & $0.34 \pm 0.03$ & - & 2 & $0.14 \pm 0.01$ & - & - \\
\hline TF-1Hi & Overexpressed IL3R $\alpha$ & 7 & $0.14 \pm 0.02$ & $<0.0001$ & 6 & $0.065 \pm 0.006$ & 0.003 & 0.004 \\
\hline $\cos$ & $\mathrm{IL} 3 \mathrm{R} \alpha+\beta \mathrm{c}$ & 15 & $0.48 \pm 0.05$ & - & 6 & $0.43 \pm 0.09$ & - & 0.98 \\
\hline $\cos$ & $\mathrm{IL} 3 \mathrm{R} \alpha \mathrm{SP} 2+\beta \mathrm{c}$ & 10 & $7.61 \pm 0.97$ & $<0.0001$ & 6 & $2.98 \pm 0.87$ & 0.011 & 0.004 \\
\hline $\cos$ & IL3R $\alpha$ & 8 & $140.6 \pm 16.9$ & - & 6 & $17.8 \pm 5.8$ & - & $<0.0001$ \\
\hline $\cos$ & IL3R $\alpha$ SP2 & 3 & N.B. & - & 6 & N.B. & - & - \\
\hline $\cos$ & IL3R $\alpha$ C76A,C195A & & n.d. & & 4 & N.B. & - & - \\
\hline $\cos$ & IL3R $\alpha$ D197L & & n.d. & & 4 & N.B. & - & - \\
\hline
\end{tabular}

interaction, such as V201, K235 or E276, preferentially reduced wild-type IL-3 function (Supplementary Table 2) and suggests that these residues indirectly influence the K116/W116 cytokine interaction. We were surprised that many IL3R $\alpha$ NTD mutations (ie, site $1 \mathrm{~b}$ ) also preferentially reduced wild-type IL-3 function, since the IL3R $\alpha$ NTD residues are distal to site $1 \mathrm{a}$ and the interaction with IL-3 K116/W116 (Figs. 1, 3, Supplementary Table 2). These results suggest that the IL3R $\alpha$ NTD plays a more dynamic role in IL-3 recognition and function than was previously anticipated.

We were also surprised that wild-type IL-3 and IL-3 K116W were indistinguishable at stimulating the proliferation of CTLL-2 cells expressing IL3R $\alpha$ and $\beta c\left(E_{50}=0.028 \mathrm{ng} \mathrm{ml}^{-1}\right.$ and $0.018 \mathrm{ng}$ $\mathrm{ml}^{-1}$ respectively, Supplementary Table 2 ). Similarly, we observed that the binding affinity of wild-type IL-3 and IL-3 K116W to CTLL-2 cells expressing IL3R $\alpha$ and $\beta c$ was indistinguishable $\left(K_{\mathrm{D}}\right.$ $=0.34 \mathrm{nM}$ vs $0.14 \mathrm{nM}$ respectively, Table 2). Comparable binding affinities were also obtained using COS cells transiently expressing IL3R $\alpha$ and $\beta c \quad\left(K_{\mathrm{D}}=0.48 \mathrm{nM}\right.$ vs $0.43 \mathrm{nM}$ respectively, Table 2). In contrast, using the TF-1 human erythroleukaemic cell line, IL-3 K116W displayed the expected superkine behaviour with enhanced binding $\left(K_{\mathrm{D}}=0.35 \mathrm{nM}\right.$ vs $2.06 \mathrm{nM}$ respectively, Table 2) and function $\left(\mathrm{ED}_{50}=0.008 \mathrm{ng} \mathrm{ml}^{-1}\right.$ and $0.044 \mathrm{ng} \mathrm{ml}^{-1}$ respectively, Supplementary Fig. 6a) relative to wild-type IL-3. Considering that endogenous IL-3 receptor expression is typically low on primary cells compared to engineered cell lines (Supplementary Fig. 6b), we wondered if this contributed to the differences in IL-3 function. Using a TF-1 cell line engineered to overexpress IL3R $\alpha^{26}$ (Supplementary Fig. 6b), we observed that wild-type IL-3 and IL-3 K116W are functionally indistinguishable $\left(\mathrm{ED}_{50}=0.005 \mathrm{ng} \mathrm{ml}^{-1}\right.$ each, Supplementary Fig. 6a), and importantly, while wild-type IL-3 is significantly more potent (approximately ninefold) on cells expressing high levels of IL3R $\alpha$, IL-3 K116W is not. Surprisingly, wild-type IL-3 and IL$3 \mathrm{~K} 116 \mathrm{~W}$ both display comparable and enhanced binding to TF$1 \mathrm{Hi}$ cells $\left(K_{\mathrm{D}}=0.14 \mathrm{nM}\right.$ vs $0.065 \mathrm{nM}$ respectively, Table 2) perhaps reflecting differences in the IL-3 receptor in these cells. Our results indicate that the binding and function of wild-type IL-3 is positively influenced by the level of IL3R $\alpha$ expression, whereas IL-3 K116W is markedly less influenced in these cells.

Explanation for the enhanced binding of IL-3 K116W. The crystal structures of wild-type IL-3 and IL-3 K116W bound to
IL3R $\alpha$ are very similar (Fig. 4, Supplementary Fig. 2b) and yet the ligand-binding affinity for these two complexes are very different. Compared to wild-type IL-3, IL-3 K116W has enhanced binding to full-length IL3R $\alpha$ expressed on COS cells $\left(K_{\mathrm{D}}=140.6 \mathrm{nM}\right.$ vs $17.8 \mathrm{nM}$, respectively, Table 2$)$ or to soluble IL3R $\alpha\left(K_{\mathrm{D}}=510 \mathrm{nM}\right.$ vs $5.3 \mathrm{nM}$ respectively ${ }^{12}$ ). The increased receptor affinity of IL-3 $\mathrm{K} 116 \mathrm{~W}$ is due to a combination of an increased association constant and decreased dissociation constant ${ }^{12}$. Examination of the wild-type IL-3 and IL-3 K116W conformational states by MD simulations showed that IL-3 K116W was conformationally restricted compared to wild-type IL-3 (Fig. 5, Supplementary Figs. $4 \mathrm{~b}, \mathrm{c}, 7 \mathrm{a}-\mathrm{c})$. The reduced conformational flexibility of IL-3 K116W, together with the W116 side chain slotting into a site 1a pocket (Fig. 4b, d), could account for the increased association constant of the IL-3 K116W superkine.

We have previously hypothesised that the IL3R $\alpha$ NTD is a highly mobile domain as IL3R $\alpha$ can exist in open and closed conformations based on differences in NTD placement in the crystal structure $^{11}$. IL3R $\alpha$ transitioning to the closed conformation was proposed to occur upon binding of IL-3 to site 1a and through the resultant engagement of site $1 \mathrm{~b}$ in the NTD, lead to reduced IL-3 dissociation. The closed IL3R $\alpha$ conformation is very similar to the conformation observed in the binary complex crystal structures herein (Supplementary Fig. 2b). Interestingly, the smaller root-mean-square fluctuation of the cytokine NTD $\mathrm{C} \alpha$ atoms and the reduced interatomic distance between IL-3 E43-IL3R $\alpha$ K54 and IL-3 D44-IL3R $\alpha$ Y58 demonstrates that the IL-3 K116W binary complex has a reduced mobility compared to the wild-type IL-3 binary complex (Fig. 5, Supplementary Fig. 7h, i). When overlaid via the CRM (ie, D2-D3), the conformational stability of the IL3R $\alpha$ NTD is increased in the wild-type IL-3 binary complex and is most pronounced in the IL-3 K116W binary complex compared to the unliganded (ie, apo) form of IL3R $\alpha$ (Fig. 5a, b). The reduced mobility of the IL-3 K116W binary complex can also be visualised by the alignment of $10 \mathrm{~ns}$ snapshots from the binary complex MD simulations via the cytokine (left hand images in Fig. 5c, d). The conformational variability of IL3R $\alpha$ over the $100 \mathrm{~ns}$ simulation timeframe is far smaller for the IL-3 K116W than the wild-type IL-3 binary complex.

Observing the decreased conformational mobility of IL3R $\alpha$ NTD in the IL-3 K116W binary complex, we investigated the contribution of the NTD as a whole by examining IL3R $\alpha$ SP2, a 
splice variant of IL3R $\alpha$ lacking the $\mathrm{NTD}^{24}$ (Supplementary Fig. 1c) and with no measurable binding to IL-3 (Table 2). Emphasising the critical role played by the NTD, we also observed no measurable binding of IL-3 K116W to cells expressing IL3R $\alpha$ SP2 (Table 2). To assess the effect of the
NTD upon cytokine:IL3R $\alpha$ complex stability, the IL3R $\alpha$ NTD in both binary complexes was truncated and the $\mathrm{N}$-terminal residues mutated in silico to those in the published SP2 sequence (Supplementary Fig. 1c) ${ }^{24}$. When 10 ns snapshots from the 100 ns binary complex MD simulations were overlaid via the wild-

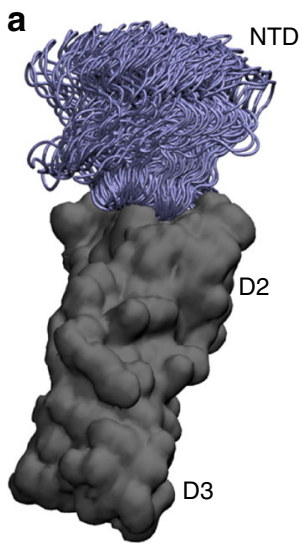

Closed IL3R $\alpha$

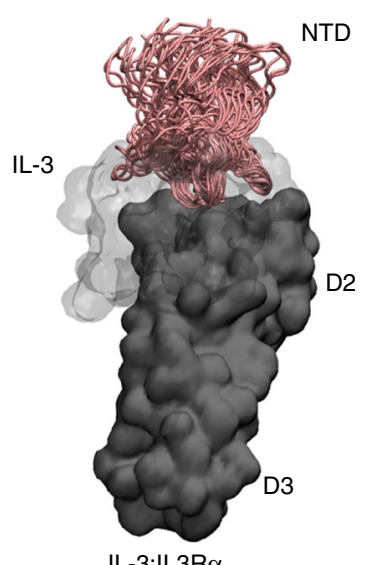

IL-3:IL3R $\alpha$

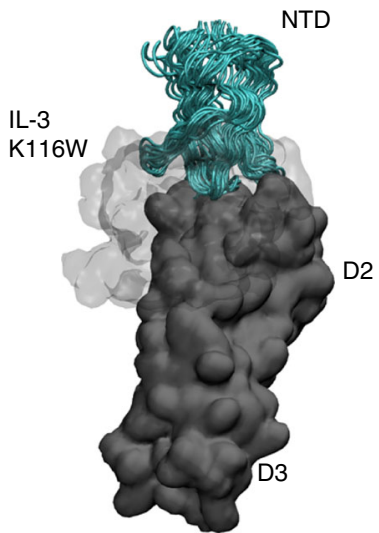

IL-3 K116W:IL3R $\alpha$
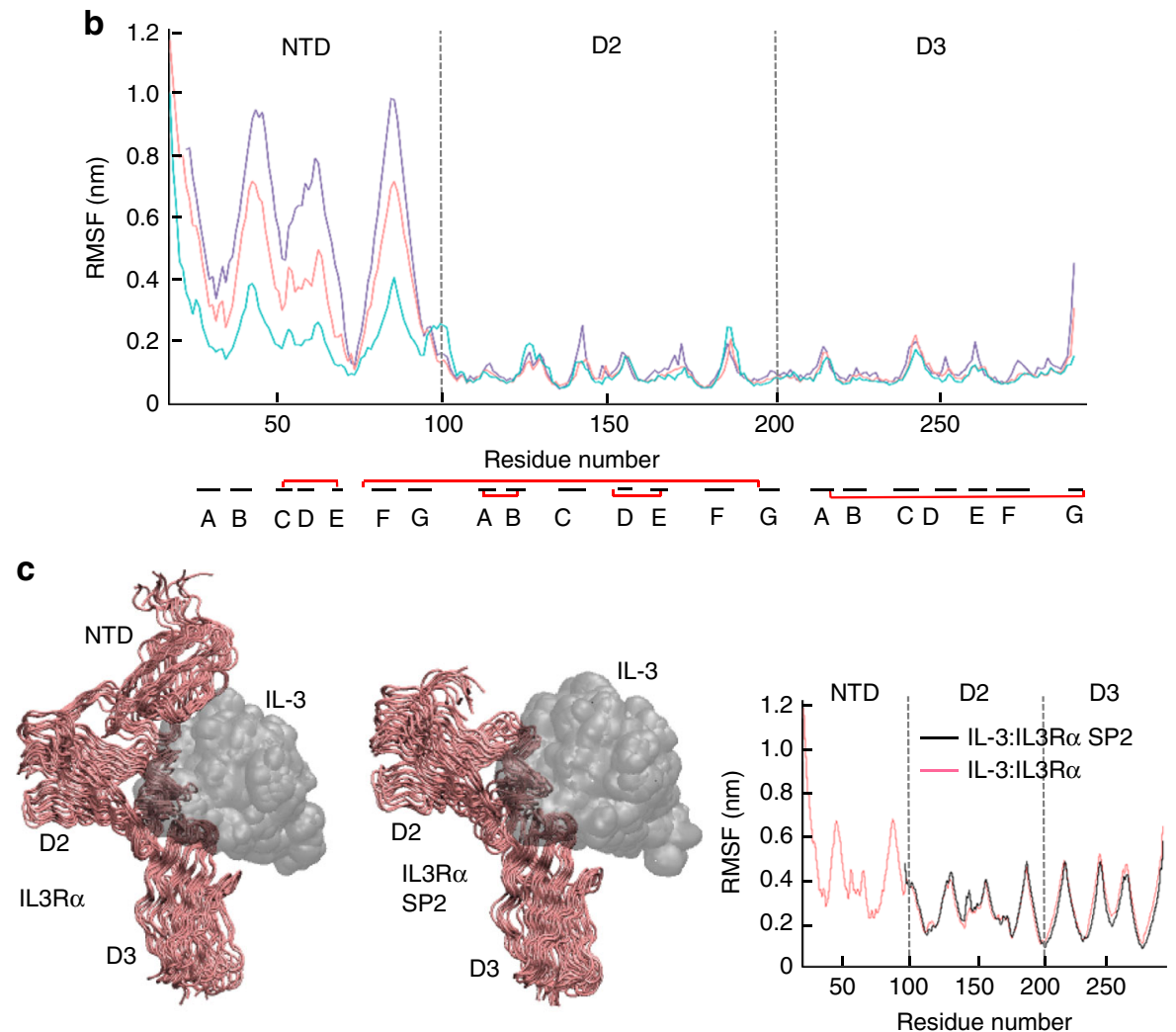

d
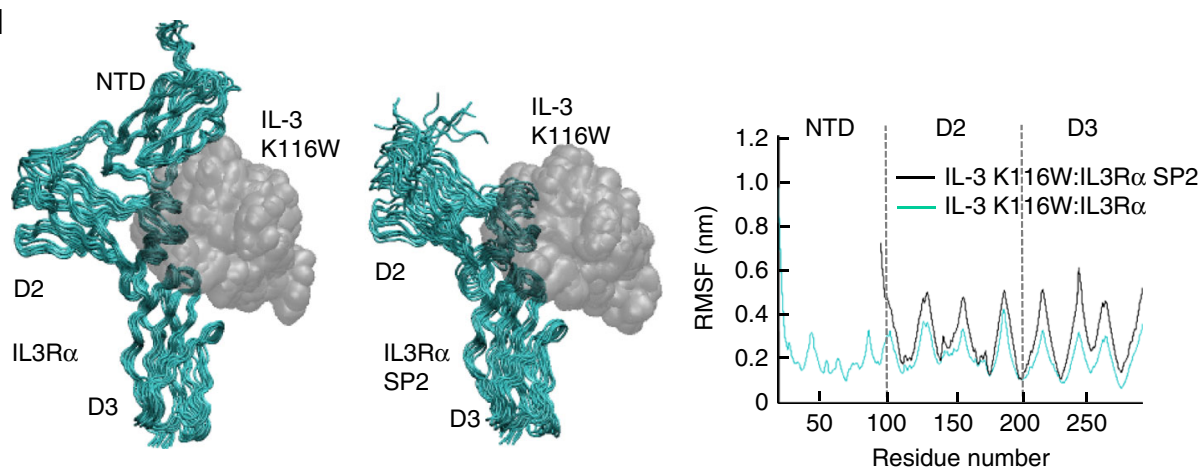
type IL-3, there was little difference in the conformational variability of wild-type or SP2 IL3R $\alpha$ (Fig. 5c). However, the stabilising role of the NTD is apparent in the simulations of the IL-3 K116W binary complexes (Fig. 5d).

NTD prevents spontaneous receptor dimerisation. We next examined what elements in the IL3R $\alpha$ NTD were important to support its correct conformation to maximise IL-3 binding and signalling. Our previous crystal structure of IL3R $\alpha$ complexed to an antibody ${ }^{11}$ and the structure of IL3R $\alpha$ complexed to IL-3 presented here (Fig. 1a) indicated that the IL3R $\alpha$ NTD domain is constrained by a disulphide bond between C76 in the NTD and C195 in D2, and by a hydrogen bond between S74 in the NTD and D197 in D2. We therefore investigated if the functional contribution of the IL3R $\alpha$ NTD was influenced by mutations that retain the NTD but perturb its presentation to IL-3. The double mutation C76A and C195A abolished the disulphide bond and was therefore predicted to produce IL3R $\alpha$ with a tethered but highly mobile NTD. Similarly, the IL3R $\alpha$ mutation D197L was predicted to fill a pocket between the NTD and D2 and, through steric interference, force IL3R $\alpha$ to adopt an open-like conformation hence also impairing site $1 \mathrm{~b}$ interactions ${ }^{11}$. MD simulations (75 ns) of the IL3R $\alpha$ C76A,C195A and IL3R $\alpha$ D197L mutants suggest an increased mobility for the NTD in both cases (Fig. 6a). Breaking the IL3R $\alpha$ NTD-D2 inter-domain disulphide bond results in the NTD twisting laterally away from the conformations adopted by the apo wild-type IL3R $\alpha$ (Fig. 6a, b, d). Increasing the bulk of residue 197 forces the NTD away from D2 and the distance between the S74-L197 C $\alpha$ atoms for the IL3R $\alpha$ D197L mutant is similar to that observed for the open apo wildtype IL3R $\alpha$ (Fig. 6a, c, e). Thus, the IL3R $\alpha$ C76A,C195A and IL3R $\alpha$ D197L mutations are both predicted to exhibit 'SP2-like' activities as the NTD is displaced (Fig. 6d, e) and no longer plays a stabilising role in cytokine binding.

We were unable to detect direct binding of IL-3 K116W to COS cells expressing the IL3R $\alpha$ C76A,C195A or IL3R $\alpha$ D197L mutants, which was in contrast to the relatively high-affinity binding we observed on cells expressing full-length IL3R $\alpha$ but comparable to the lack of direct binding we observed for the IL3R $\alpha$ SP2 isoform (Table 2). When co-expressed with $\beta c$, IL3R $\alpha$ SP2 and IL3R $\alpha$ D197L displayed significantly reduced binding affinities for wild-type IL-3 and IL-3 K116W (Table 2). For IL3R $\alpha$ C76A,C195A, there was a modest but significant reduction in binding affinity for wild-type IL-3 but not IL-3 K116W (Table 2).

To examine the functional capacity of the constrained NTD mutants, we examined the response of mouse FDH cells expressing these receptors. Cells co-expressing $\beta c$ and IL3R $\alpha$ SP2, IL3R $\alpha$ C76A,C195A and IL3R $\alpha$ D197L proliferated in response to wild-type IL-3 and IL-3 K116W stimulation but with reduced potency compared to cells expressing full-length IL3R $\alpha$
$\left(\mathrm{ED}_{50}=0.29 \mathrm{ng} \mathrm{ml}^{-1}\right.$ and $0.37 \mathrm{ng} \mathrm{ml}^{-1}$ respectively, Table 3). Cells expressing IL3R $\alpha$ SP2 and IL3R $\alpha$ C76A,C195A had comparable functional responses to wild-type IL-3 $\left(\mathrm{ED}_{50}=6.94 \mathrm{ng} \mathrm{ml}^{-1}\right.$ and $12.17 \mathrm{ng} \mathrm{ml}^{-1}$ respectively, Table 3$)$ and IL-3 K116W $\left(\mathrm{ED}_{50}=\right.$ $2.19 \mathrm{ng} \mathrm{ml}^{-1}$ and $2.51 \mathrm{ng} \mathrm{ml}^{-1}$ respectively, Table 3). In contrast, cells expressing IL3R $\alpha$ D197L displayed a modest reduction in cell proliferation to both wild-type IL-3 and IL-3 K116W $\left(\mathrm{ED}_{50}=\right.$ $1.71 \mathrm{ng} \mathrm{ml}^{-1}$ and $0.60 \mathrm{ng} \mathrm{ml}^{-1}$ respectively, Table 3). Therefore, elimination of the $\mathrm{C} 76-\mathrm{C} 195$ disulphide bond that constrains NTD mobility or substitutions at D197 that prevent proper engagement of the NTD, produce full-length IL3R $\alpha$ that has functional similarities with IL3R $\alpha$ SP2. Modest improvements in binding affinity (Table 2) and potency (Table 3) for IL-3 K116W relative to wild-type IL-3 are consistent with IL-3 K116W having an enhanced interaction with IL3R $\alpha$ D2-D3. Collectively, these results demonstrate that the NTD interaction with IL-3 is exquisitely sensitive to conformational changes.

We next examined whether the IL3R $\alpha$ NTD was important for receptor heterodimerisation and tyrosine phosphorylation as a measure of receptor signalling. Using HEK293-T cells stably expressing $\beta c$ and transiently co-expressing IL3R $\alpha$ SP2, IL3R $\alpha$ C76A,C195A and IL3R $\alpha$ D197L (Supplementary Fig. 8), we observed IL-3-dependent, co-immunoprecipitation (IP) of Flagtagged IL3R $\alpha$ following $\beta c$ IP that was associated with tyrosine phosphorylation of $\beta c$ (Fig. 6f). Surprisingly, deletion of the NTD was associated with constitutive association of IL3R $\alpha$ SP2 with $\beta c$ but the retention of IL-3-dependent tyrosine phosphorylation of $\beta c$ (Fig. 6f). Consistent with their SP2-like properties, IL3R $\alpha$ C76A,C195A, and to a lesser extent, IL3R $\alpha$ D197L displayed constitutive association with $\beta c$ but retained IL-3-dependent tyrosine phosphorylation of $\beta c$ (Fig. 6f). These data strongly suggest that a naturally constrained IL3R $\alpha$ NTD prevents spontaneous IL3R $\alpha$ heterodimerisation with $\beta c$ and could potentially protect cells from unregulated signalling.

\section{Discussion}

We report here the three-dimensional atomic structures of the IL3 receptor $\alpha$-subunit in complex with IL-3 and with the IL-3 K116W superkine, revealing the key interactions between receptor and cytokine that compose site 1 , the first and major recognition step that initiates signalling in this family of receptors. Of particular significance, is the finding through extensive analyses by functional and MD simulation approaches that the NTD of IL3R $\alpha$ exhibits exquisite flexibility and a dynamic transition from a high to a low mobility state upon cytokine binding that leads to optimal ligand recognition, receptor dimerisation and signalling. Since the NTD is also a feature of other members of the type I cytokine receptor family, the results are likely to have general implications for our understanding of how the NTDs function in receptor assembly and signalling in other cytokine receptors.

Fig. 5 Ensemble of IL3R $\alpha$ MD simulations in isolation and in complex with wild-type IL-3 and IL-3 K116W. a Snapshots from $75 \mathrm{~ns}$ (unliganded/apo IL3R $\alpha$ ) and 100 ns (binary complexes) MD simulations overlaid via the IL3R $\alpha$ CRM (ie, D2-D3). The mobility of the NTD progressively decreases when going from the unliganded/apo IL3R $\alpha$ (purple NTD, left), to the wild-type IL-3 binary complex (pink NTD, middle) and then to the IL-3 K116W binary complex (green NTD, right). The IL3R $\alpha$ CRM is shown as a dark grey molecular surface, the NTD is shown as a cartoon and the cytokine is depicted as a light grey molecular surface. $\mathbf{b}$ Root-mean-square fluctuation (RMSF) of the C $\alpha$ atoms in IL3R $\alpha$ provides a measure of atom mobility around its average position during the simulation. The data demonstrates that IL-3 K116W stabilises the NTD in the binary complex. Colour scheme and IL3R $\alpha$ alignment as for a. The location of the $\beta$-strands (black lines) and the disulphide bonds (red lines) are shown below the X-axis. c Comparison of full-length IL3R $\alpha$ vs IL3R $\alpha$ SP2 mobility in the wild-type IL-3 binary complex. Snapshots from the $100 \mathrm{~ns} \mathrm{MD} \mathrm{simulations} \mathrm{overlaid} \mathrm{via} \mathrm{the} \mathrm{cytokine} \mathrm{(grey} \mathrm{molecular} \mathrm{surface).} \mathrm{IL3R} \alpha$ shown as a pink cartoon. Loss of the NTD has little impact upon the conformational mobility of the $\alpha$-subunit. d Comparison of full-length IL3R $\alpha$ vs IL3R $\alpha$ SP2 mobility in the IL-3 K116W binary complex. Snapshots from the 100 ns MD simulations overlaid via the cytokine (grey molecular surface). IL3R $\alpha$ shown as a green cartoon. Loss of the NTD has a visible negative impact upon the conformational mobility of the $\alpha$-subunit and it now resembles the wild-type IL3 system shown in $\mathbf{c}$ 
a

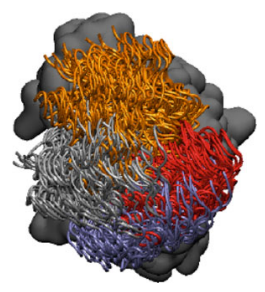

b

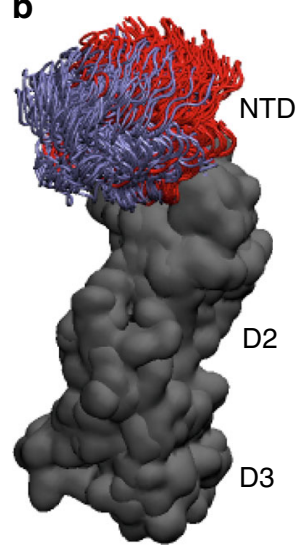

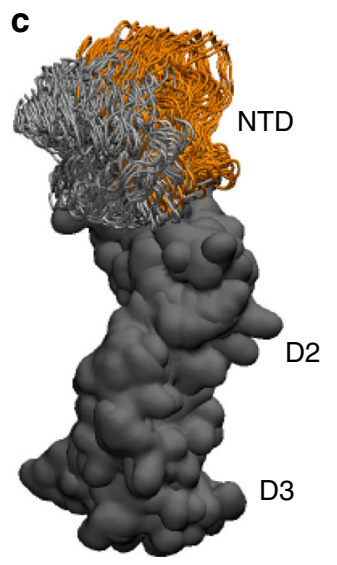

C

e

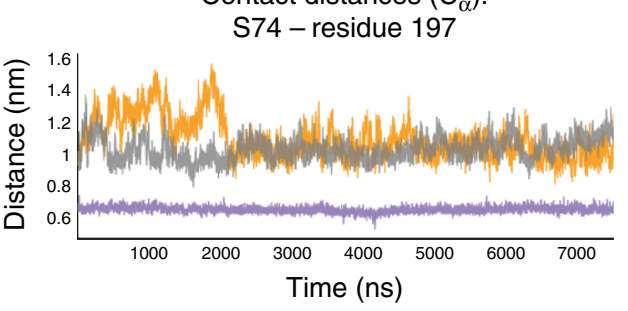

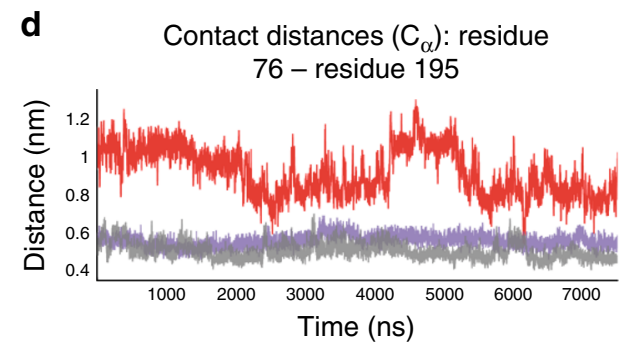

76 - residue 195

f
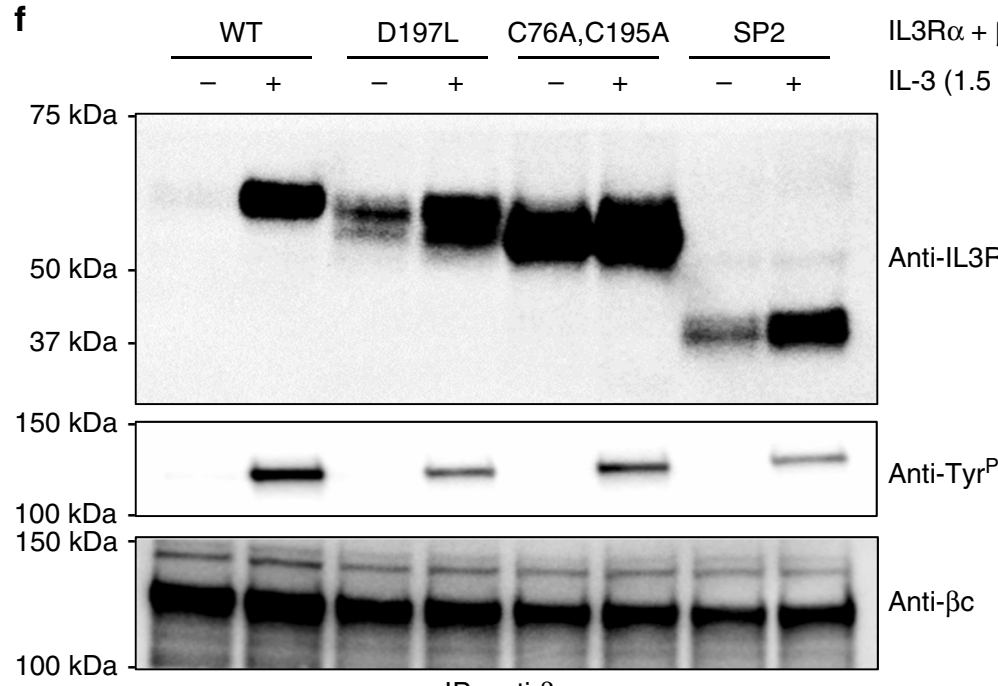

IL $3 R \alpha+\beta c$

$\mathrm{IL}-3\left(1.5 \mu \mathrm{g} \mathrm{ml}^{-1}\right)$

IP: anti- $\beta c$

Fig. 6 IL3R $\alpha$ mutations that perturb NTD placement also promote spontaneous receptor heterodimerisation. Snapshots from apo IL3R $\alpha$ MD simulations (75 ns wild-type, $100 \mathrm{~ns}$ mutants) overlaid via the IL3R $\alpha$ CRM. Simulations initiated from both the closed and open wild-type apo IL3R $\alpha$ conformations. a When looking down onto the NTD, the range of conformations sampled by the apo wild-type IL3R $\alpha$ closed (purple) and open (grey) starting conformations, the C76A,C195A mutant (red) and D197L mutant (orange) can be clearly observed. $\mathbf{b}$ In the IL3R $\alpha$ C76A, C195A mutant, the NTD twists away from those conformations adopted by the closed (or open, not shown) apo wild-type IL3R $\alpha$. $\mathbf{c}$ In the IL3R $\alpha$ D197L mutant, the NTD is also forced away from D2 when compared to the conformations adopted by the open (or closed, not shown) apo wild-type IL3R $\alpha$. a-c the IL3R $\alpha$ CRM is depicted as a dark grey molecular surface and the NTD as a cartoon. View in $\mathbf{b}$ and $\mathbf{c}$ is a rotation of $+90^{\circ}$ about the $X$-axis from that shown in $\mathbf{a}$. $\mathbf{d}$ Distance between the $C \alpha$ atoms of the C76-C195 inter-domain disulphide bond in the closed (purple) and open (grey) starting IL3R $\alpha$ conformations compared to that in the C76A, C195A mutant (red). The greater distance between the NTD and D2 for the C76A,C195A mutant is consistent with the reduced binding affinity for IL-3. e Distance between the C $\alpha$ atoms of S74-D197 in the closed (purple) and open (grey) starting conformations compared to that in the IL3R $\alpha 197 \mathrm{~L}$ mutant (orange). In the closed wild-type IL3R $\alpha$ conformation, S74 (NTD) and D197 (D2) form an inter-domain hydrogen bond, whereas the distance is too great in the open conformation. The leucine side chain of the D197L mutant keeps the NTD and D2 apart in an open-like conformation. $\mathbf{f}$ HEK293T cells stably expressing $\beta c$ were transfected with either wild-type (WT), D197L, C76A,C195A or SP2 forms of Flag-tagged IL3R $\alpha$ and stimulated with $1.5 \mu g$ ml ${ }^{-1}$ IL-3 for $45 \mathrm{~min}$ at $4^{\circ} \mathrm{C}$. Cell lysates were immunoprecipitated with the $\beta c$ antibody BION-1. Immunoprecipitates were immunoblotted with antibodies for IL3R $\alpha$ (anti-IL3R $\alpha$ ), phosphotyrosine (anti-Tyr ${ }^{\mathrm{P}}$ ) or total $\beta c($ anti- $\beta c)$ 
Table 3 Enhanced site 1a interactions do not fully compensate for perturbations of functional interactions through the NTD

\begin{tabular}{|c|c|c|c|c|c|c|c|}
\hline \multirow[t]{2}{*}{ IL3R $\alpha$} & \multicolumn{3}{|c|}{ IL-3 } & \multicolumn{3}{|c|}{ IL-3 K116W } & \multirow[t]{2}{*}{$P$} \\
\hline & $n$ & $\mathrm{ED}_{50}\left(\mathrm{ng} \mathrm{ml}^{-1}\right)$ & $p$ & $n$ & $E D_{50}\left(n ~ m l^{-1}\right)$ & $p$ & \\
\hline Wild type & 6 & $0.29 \pm 0.11$ & - & 6 & $0.37 \pm 0.10$ & - & 0.58 \\
\hline C76A,C195A & 3 & $12.17 \pm 2.02$ & 0.0002 & 3 & $2.51 \pm 0.49$ & 0.002 & 0.01 \\
\hline D197L & 3 & $1.71 \pm 0.08$ & 0.0002 & 3 & $0.60 \pm 0.13$ & 0.27 & 0.002 \\
\hline
\end{tabular}

Functional activity of IL3R $\alpha$ mutants was measured in IL-3 or IL-3 K116W dose-response proliferation assays using FDH cell lines transduced to co-express the $\beta c$ subunit with either wild-type or mutant forms of IL3R $\alpha$. Half-maximal responses $\left(E_{50}\right)$ were calculated from each experiment and averaged. Errors represent SEM from the indicated number $(n)$ of experiments. Statistical significance of functional response between wild-type IL3R $\alpha$ and the mutant forms of IL3R $\alpha(p)$ or between IL-3 and IL-3 K116W (P) was determined using a two-tailed paired $t$ test. Related to Figs. 5, 6, Supplementary Fig. 8 and Table 2

A comparison of the IL-3 binary complexes showed that the IL3R $\alpha$ site 1a interaction involves the elbow region of D2 and D3 (Fig. 1a, c) that follows the paradigm established by the growth hormone receptor ${ }^{25}$. In both complexes, a large interface was seen with mutations of Q178, V201, Q204 and E276 in IL3R $\alpha$ having a significant effect upon wild-type IL-3 and IL-3 K116W signalling (Supplementary Table 2). In contrast, mutations of N233 and Y279 in IL3R $\alpha$ had a significant effect upon wild-type IL-3 but not IL-3 K116W signalling indicating an altered role for interactions through these residues for IL-3 K116W. The major difference observed between the two complexes was the side chain of the superkine's W116 that inserts itself in a groove formed by Q204 and Y279 of IL3R $\alpha$ (Fig. 4), with mutation of Q204 reducing both wild-type IL-3 and IL-3 K116W signalling (Supplementary Table 2).

Both IL-3 binary complexes have a very similar site $1 \mathrm{~b}$ interface, yet they display important functional differences. The Dstrand of the IL3R $\alpha$ NTD shows a close interaction with the AB loop and CD loop of IL-3 (Fig. 1b). This helps to explain why mutations of IL-3 E43 or D44 in the $\alpha 2$-helix of the AB loop disrupt IL-3 function ${ }^{16}$, whereas mutation of adjacent residues in the $\alpha 2$-helix including G42, Q45 and D46 enhances IL-3 binding to IL3R $\alpha$ as well as IL-3 function ${ }^{12,16}$. Furthermore, mutagenesis of IL3R $\alpha$ NTD residues K54, Y58 or G71 abolishes direct IL-3 binding ${ }^{11}$. The IL3R $\alpha$ NTD EF loop is stabilised by an interaction with the NTD B-strand residue Q37, which makes main-chain contacts through its side chain with I73, and carbonyl interactions with Q69 and F70. Mutation of IL3R $\alpha$ Q37 also reduces IL-3 binding $^{11}$, presumably as a result of changes to the NTD EF loop conformation. Despite marked differences in the specific interactions that comprise site $1 \mathrm{~b}$, the surface area between cytokine and IL $3 R \alpha$ at the site $1 \mathrm{~b}$ interface is relatively similar among the related receptor complexes (34\% in IL3R $\alpha, 42 \%$ in GMR $\alpha$ (PDB ID: $4 \mathrm{RS} 1^{11}$ ), $64 \%$ in IL5R $\alpha$ (PDB ID: $3 \mathrm{VA2} 2^{21}$ ), $47 \%$ in IL13R $\alpha 1$ (PDB ID: $3 \mathrm{BPO}^{22}$ ) and $34 \%$ in IL13R $\alpha 2$ (PDB ID: $3 \mathrm{LB}^{23}$ )) (Fig. 2, Supplementary Fig. 2; see additional detail in Supplementary Note 1) suggesting that it may be serving similar functions in these other receptors. Interestingly, despite there being essentially no difference in the way that IL-3 and IL-3 K116W interacted with IL $3 R \alpha$ through site $1 \mathrm{~b}$, there were some crucial differences in how mutation of many of these residues selectively impaired the wild-type IL-3 function. Unlike IL3R $\alpha$ interactions with IL-3, the interaction with IL-3 K116W was not markedly affected by mutations of K54, Y58 or G71 in the NTD (Fig. 3, Supplementary Table 2) indicating that the K116W mutation has stabilised the interaction with IL3R $\alpha$ through distal effects on the NTD.

In the published structure of IL3R $\alpha$ bound to the blocking antibody CSL362, we found the NTD in two distinct structural forms, an open and a closed conformation ${ }^{11}$ that led us to hypothesise that the IL3R $\alpha$ NTD may be a highly mobile domain. We now show by MD simulations that indeed the NTD is highly mobile and that the presence of IL-3 stabilises the NTD in the binary complex. The largest effect was observed for IL-3 K116W, which restricts the conformational flexibility of the binary complex (Figs. 4, 5a, b), probably as a result of W116 inducing a distal effect that stabilises the engagement with the NTD thus restricting domain mobility. This high mobility may be a general feature of the NTD of the type I cytokine receptor family and a major reason for the difficulty in their crystallisation, particularly in the ligand-free state. The flexibility and high mobility of the NTD may facilitate locking of the cytokine upon binding to site $1 \mathrm{a}$ and the appropriate presentation of the cytokine: $\alpha$-subunit complex to the signalling subunit. Similarly, in the interferon (IFN) receptor, IFNAR1, conformational flexibility has been observed in the IFN-bound and unliganded/apo structures. Comparison of apo IFNAR1 with its IFN-bound form revealed a large spatial rearrangement in the four extracellular subdomains $(\mathrm{SD} 1-\mathrm{SD} 4)^{27}$. Although the IFN ligand binds largely to the hinge region between the IFNAR1 SD2 and SD3 domains, the NTD of IFNAR1 (ie, SD1) moves downwards $\sim 10 \AA$ to interact with the IFN ligand, further securing it to IFNAR1. The high mobility of IFNAR1 is required for optimal ligand binding and the formation of a ternary complex that then initiates trans-phosphorylation between JAK1 and TYK $2{ }^{28}$.

The importance of a conformationally restricted IL3R $\alpha$ NTD is further underscored by mutagenesis of the disulphide bond between the NTD and D2 and of the aspartic acid at position 197. These mutations abolished direct binding of IL-3 K116W and reduced high-affinity binding and signalling in the presence of $\beta \mathrm{c}$ (Tables 2, 3). Interestingly, mutations that perturb the position of the NTD, or that completely delete the NTD, are associated with spontaneous heterodimerisation of IL3R $\alpha$ with $\beta c$, although surprisingly, this spontaneous dimerisation does not result in receptor tyrosine phosphorylation (Fig. 6f). These observations are comparable with reports of preformed but non-signalling dimers of receptors for growth hormone ${ }^{29}$ and erythropoietin ${ }^{30}$ that signal following ligand binding-induced conformational changes in the receptor complex. In an analogous manner, the architecture of the NTD-deficient or NTD-displaced IL3R $\alpha$ may promote association with $\beta c$ to form inert IL3R $\alpha: \beta c$ complexes that only signal following conformational changes triggered by the binding of IL-3. While tyrosine phosphorylation of the $\beta \mathrm{c}$ receptor was strictly dependent upon IL-3 stimulation, other forms of receptor signalling could be associated with spontaneous heterodimerisation of IL3R $\alpha$ and $\beta c$. Additionally, in cells expressing wild-type IL $3 \mathrm{R} \alpha$, the NTD may serve a protective role by preventing signalling from receptor association arising in the absence of ligand.

The relatively high expression of IL3R $\alpha$ in a number of leukaemias has presented a window of opportunity to develop new therapeutic approaches directed to IL $3 \mathrm{R} \alpha$ such as monoclonal antibodies (MAbs) ${ }^{8,31,32}$, T cells engineered to express chimeric antigen receptors (CAR $\mathrm{T}$ cells) ${ }^{33}$ and toxin conjugates with IL- 
$3^{34-37}$. The latter was originally only partially successful as half the samples proved resistant to the fusion protein ${ }^{38}$. The potency of the toxin-IL-3 fusion protein was improved dramatically by introducing the K116W substitution into the IL-3 portion ${ }^{38-40}$. The insights described in our work explain the potency of the IL$3 \mathrm{~K} 116 \mathrm{~W}$ superkine and suggest new avenues for further protein engineering of even more potent superkines.

In comparing the effects of IL3R $\alpha$ mutations on IL-3 and IL-3 K116W binding and signalling, we noted that for cells expressing endogenous IL-3 receptors (such as TF-1 cells) there is, as previously reported by us and others, a sixfold increased potency of the IL-3 superkine (Table 2, Supplementary Fig. 6a). However, in cells overexpressing IL3R $\alpha$ (such as engineered CTLL-2 or FDH cell lines, TF-1Hi cells and COS cells), this difference was diminished or absent (Fig. 3, Supplementary Fig. 6a, Tables 2, 3, Supplementary Table 2). The observation that IL3R $\alpha$ expression levels vary widely among different primary cell populations ${ }^{41}$ suggests the likelihood of differences in IL-3 sensitivity among these cell populations. This may also have implications in AML where the AML leukaemic stem/progenitor cells express elevated levels of IL $3 R^{7,8}$, but may also indicate differences in the composition or form of the endogenous IL-3 receptor. For instance, it is known that transcripts for the IL3R $\alpha$ SP2 splice variant are expressed by TF- 1 cells ${ }^{24}$ and this could contribute to reduced IL- 3 binding and function compared to cells only expressing full-length IL3R $\alpha$.

In summary, our results highlight the unique dynamics and role of the NTD in IL-3 signalling and suggest the purpose of its conservation among members of the type I cytokine receptor family.

\section{Methods}

Production of recombinant IL-3:sIL3R $\alpha$ binary complexes. We prepared wildtype IL-3 and IL-3 K116W binary receptor complexes using partially glycosylated variants of the IL3R $\alpha$ extracellular domain and truncated wild-type IL-3 or IL-3 $\mathrm{K} 116 \mathrm{~W}$. DNA fragments encoding soluble IL3R $\alpha \Delta \mathrm{N} 5$ (sIL3R $\alpha$; residues L20-S307 of the expressed peptide with the N212Q mutation) or soluble $\beta c \Delta \mathrm{N} 3$ (s $\beta c$; residues E25-S438 of the expressed peptide with the N346Q mutation) were cloned into the $\mathrm{pFastBac1}$ vector (Invitrogen). The resulting plasmids were transformed into the DH10Bac Escherichia coli strain from which recombinant bacmid DNA was isolated and transfected into Sf9 insect cells (ATCC CRL-1711) using Cellfectin reagent (Invitrogen). Following three rounds of recombinant virus propagation, large-scale expression was performed by infection of Sf9 cells grown in Sf-900 II serum-free medium (Life Technologies). The supernatant was collected after 5 days and recombinant proteins affinity-purified using HiTrap NHS-activated HP columns (GE Healthcare) coupled to the IL3R $\alpha$-specific MAb $95^{42}$ or the $\beta c$-specific MAb BION- $1^{43}$. Bound protein was eluted using $500 \mathrm{mM} \mathrm{NaCl}, 50 \mathrm{mM}$ phosphoric acid $\mathrm{pH} 2.4$ and the eluted protein concentrated and further purified by preparative size exclusion chromatography (SEC) using a Superdex 200 column $\left(26 \times 600 \mathrm{~mm}\right.$, GE Healthcare) operated at $2 \mathrm{ml} \mathrm{min}^{-1}$ with $150 \mathrm{mM} \mathrm{NaCl}, 50 \mathrm{mM}$ sodium phosphate $\mathrm{pH} 7.0$ as running buffer ${ }^{44}$. Binding and functional studies with wild-type IL-3 demonstrate that the N212Q mutation has no impact on the function of full-length IL3R $\alpha$ (Supplementary Fig. 3c, d). Wild-type or K116W versions of human IL-3, comprising residues W13 to Q125 of the mature peptide with a W13Y mutation and a GAMGS N-terminal tail arising from the expression plasmid, were purified from E. coli ${ }^{45}$. BL21 CodonPlus (DE3)-RIPL cells transformed with pETNusH expression vectors encoding Nus-6xHis-TEV:IL-3 fusion proteins, were cultured to an $\mathrm{OD}_{600}$ of 0.6 and induced with $0.1 \mathrm{mM}$ isopropyl $\beta$ D-1-thiogalactopyranoside (IPTG) at $23^{\circ} \mathrm{C}$ for $16 \mathrm{~h}$. Cells were lysed and Nus6xHis-TEV:IL-3 fusion proteins affinity-purified using HisTrap FF columns (GE Healthcare). Fusion proteins were concentrated, dialysed against $50 \mathrm{mM}$ Tris- $\mathrm{HCl}$ $\mathrm{pH} 8.0$, adjusted to $1 \mathrm{mM}$ dithiothreitol, $0.5 \mathrm{mM}$ EDTA and incubated with TEV protease at $23{ }^{\circ} \mathrm{C}$ for $16 \mathrm{~h}$. IL-3 was purified from the TEV digest by preparative SEC then adjusted to $0.1 \% \mathrm{v} \mathrm{v}^{-1}$ trifluoroacetic acid, $1 \% \mathrm{v} \mathrm{v}^{-1}$ acetic acid and further purified by reversed-phase chromatography using an Aquapore RP300 reversed-phase column $(4.6 \times 100 \mathrm{~mm}$, PerkinElmer) equilibrated in buffer A $(0.1 \%$ $\mathrm{v} \mathrm{v}^{-1}$ trifluoroacetic acid) and eluted using a linear gradient from 0 to $100 \%$ buffer $\mathrm{B}\left(0.085 \% \mathrm{v} \mathrm{v}^{-1}\right.$ trifluoroacetic acid in acetonitrile). Fractions containing purified hIL-3 were lyophilised, resuspended in PBS and sterilised using Corning Spin-X filters (Sigma-Aldrich). We, and others, have previously demonstrated that compared to full-length recombinant IL-3, the truncated IL-3 has equivalent bioactivity ${ }^{45}$

A binary complex consisting of wild-type IL-3 and sIL3R $\alpha \Delta \mathrm{N} 5$ was isolated by SEC of a 2:1.5:1 molar ratio mixture of IL-3:sIL3R $\alpha \Delta \mathrm{N} 5: s \beta c \Delta \mathrm{N} 3$ (Supplementary Fig. 1a). Using a similar strategy, a binary complex consisting of IL-3 K116W and
sIL3R $\alpha \Delta$ N5 was isolated by SEC of a 1.6:1 molar ratio mixture of IL-3 K116W: sIL3R $\alpha$ N5 (Supplementary Fig. 1b).

Analysis of sIL3R $\alpha \mathbf{N}$-linked glycosylation mutants. Soluble IL3R $\alpha$ complementary DNA (cDNA) with a C-terminal 6xHis tag (sIL3R $\alpha-6 \mathrm{xHis})$ was cloned into the pcDNA3.1 expression vector (Invitrogen) and asparagine to glutamine mutations generated by PCR at the six predicted N-linked glycosylation sites. Freestyle $^{\mathrm{TM}} 293$ suspension cells (Invitrogen) were transiently transfected with the pcDNA:sIL3R $\alpha-6 x H i s$ plasmids ${ }^{11}$ and the resulting supernatants immunoblotted with $0.9 \mu \mathrm{g} \mathrm{ml}^{-1}$ of the anti-IL3R $\alpha \mathrm{MAb}, 7 \mathrm{G}^{42}$ and $1 \mu \mathrm{g} \mathrm{ml}^{-1}$ of goat-anti-mouse IgG - fluorescein isothiocyanate (IgG-FITC (Thermofisher 62-6511)) or $0.25 \mu \mathrm{g} \mathrm{ml}$ -1 of FITC-conjugated anti-His tag antibody (Anti-6xHis Genescript cat no. A01620).

Structure determination of IL-3:IL3R $\alpha$ binary complexes. Crystallisation was performed using the hanging drop vapour diffusion method at $4{ }^{\circ} \mathrm{C}$. Crystallisation trials of IL-3 K116W:IL3R $\alpha$ were set up using the protein crystallisation screen $\mathrm{JCSG}^{46}$ at the CSIRO C 3 crystallisation facility (http://crystal.csiro.au) with the protein at a concentration of $12 \mathrm{mg} \mathrm{m}^{-1}$. Protein of $0.15 \mu \mathrm{l}$ was mixed with $0.15 \mu \mathrm{l}$ of crystallisation reagent and hung over $50 \mu \mathrm{l}$ of reservoir solution. Tetragonal crystals appeared after 5 days in $18-23 \%\left(\mathrm{w} \mathrm{v}^{-1}\right)$ PEG $8000,200 \mathrm{mM} \mathrm{NaCl}$ and 100 $\mathrm{mM}$ citrate-phosphate buffer $\mathrm{pH}$ range $4.6-5$. After screening around this condition, the best crystals were obtained in $20 \%$ PEG $8000,200 \mathrm{mM} \mathrm{NaCl}$ and $100 \mathrm{mM}$ citrate-phosphate buffer $\mathrm{pH} 4.8$. The crystals diffracted to a resolution of $2.4 \AA$ in the hexagonal space group $P 6_{1}$. The space group and cell dimensions were consistent with there being one complex in the asymmetric unit giving a Matthews coefficient of $3.0 \mathrm{Da} \AA^{-3}$ and an estimated solvent content of $65 \%$. Before data collection the crystals were dipped consecutively into $5 \%\left(\mathrm{v} \mathrm{v}^{-1}\right), 10 \%\left(\mathrm{v} \mathrm{v}^{-1}\right), 15 \%$ $\left(\mathrm{v} \mathrm{v}^{-1}\right)$ cryomix (8.4\% each of ethylene glycol, glycerol, 2-methyl-2,4-pentanediol, sorbitol, PEG 400, and sucrose) in crystallisation buffer.

Data were collected on the MX2 beamline at the Australian Synchrotron (Clayton, Victoria). Data collection was controlled using Blue-Ice software ${ }^{47}$ and processed with the HKL2000 suite ${ }^{48}$. The structures were solved with the program Phaser ${ }^{49}$ initially trialling a range of search models including IL3R $\alpha$ open and closed conformations (PDB ID: 4JZJ), IL13R $\alpha 1$ (PDB ID: 3BPO), IL13R 22 (PDB ID: 3LB6), IL5R $\alpha$ (PDB IDs: 3QT2, 3VA2), and the partial GMR $\alpha$ structure from the ternary GM-CSF complex (PDB ID: 3CXE). The final model used for molecular replacement for the IL-3 K116W binary complex was the nuclear magnetic resonance structure for IL-3 (PDB ID: 1JLI) and the IL13R 22 receptor (PDB ID: 3BL6) as separate search components. The results from Phaser gave a final translation function $Z$-score of 9.6. One IL-3 K116W:IL3R $\alpha$ complex was found in the asymmetric unit. Loop regions were manually built in place using $\mathrm{COOT}^{50}$ and subsequent rounds of refinement using REFMAC ${ }^{51}$. Residues spanning 27-293 of IL3R $\alpha$ and 12-123 in IL-3 were identified with clear and unambiguous electron density observed at the IL-3:IL3R $\alpha$ interface. Electron density was not observed for residues $42-50$ and $86-90$ in IL3R $\alpha$, which are located in flexible loop regions. Electron density representing glycan moieties was observed at glycosylation sites $\mathrm{N} 80$ and N218 in IL3R $\alpha$. The stereochemical quality of the final model correlates well with structures at similar resolutions with only $0.5 \%$ of residues in the disallowed regions of the Ramachandran plot. Other stereochemical parameters were are all better than the allowed ranges defined by PROCHECK ${ }^{52}$. Data and refinement statistics are listed in Table 1 and stereo images of portions of the electron density maps in Supplementary Fig. 10. The PISA (Protein Interfaces, Surfaces and Assemblies) server http://www.ebi.ac.uk/msd-srv/prot_int/pistart. $\mathrm{html}$ was used for all protein-ligand surface interaction calculations.

We have previously tried exhaustive crystallisation screening and construct optimisation of wild-type IL-3:IL3R $\alpha$ for many years without success. Our results described here provide a possible explanation: the mobility of the NTD inhibits crystallisation. Crystals of wild-type IL-3:IL3R $\alpha$ did not grow under the same condition as IL-3 K116W:IL3R $\alpha$; we were only able to crystallise IL-3:IL3R $\alpha$ when microcrystals of IL-3 K116W:IL3R $\alpha$ were used to seed crystals of wild-type IL-3: IL3R $\alpha$. Drops comprised $0.2 \mu \mathrm{l}$ of seed solution, $0.4 \mu \mathrm{l}$ of protein and $0.4 \mu \mathrm{l}$ of crystallisation reagent and hung over $1 \mathrm{ml}$ of reservoir solution. Tooth-shaped crystals appeared after 5 days in 20-22\% PEG $8000,200 \mathrm{mM} \mathrm{NaCl}$ and $100 \mathrm{mM}$ citrate-phosphate buffer $\mathrm{pH}$ range 4.8-5. The crystals diffracted to a resolution of $2.7 \AA$ in the hexagonal space group $P 6_{5} 22$. The space group and cell dimensions were consistent with there being two complexes in the asymmetric unit giving a Matthews coefficient of $2.8 \mathrm{Da} \AA^{-3}$ and an estimated solvent content of $58 \%$. Before data collection, the crystals were cryoprotected as described for the IL-3 K116W: IL3R $\alpha$ crystals.

The structure of wild-type IL-3:IL3R $\alpha$ was solved using the refined model of IL3 K116W:IL3R $\alpha$ and the programs described above. The results from Phaser gave a final translation function $Z$-score of 47.1. Two wild-type IL-3:IL3R $\alpha$ complexes were found in the asymmetric unit. Building and refinement was completed as described above. Residues spanning 25-294 of IL3R $\alpha$ and 13-121 in wild-type IL-3 were identified with clear and unambiguous electron density observed at the IL-3: IL3R $\alpha$ interface. Chains $G$ and I were the most complete of the two copies, however electron density was not observed for residues 48 and 143-146 in IL3R $\alpha$, which are all located in flexible loop regions. Electron density representing glycan moieties was observed at glycosylation sites N80, N109 and N218 in IL3R $\alpha$. The 
stereochemical quality of the final model correlates well with structures at similar resolutions with only $0.3 \%$ of residues in the disallowed regions of the Ramachandran plot. Other stereochemical parameters were all better than the allowed ranges defined by PROCHECK ${ }^{52}$. Data and refinement statistics are listed in Table 1.

Molecular dynamics simulations. Fully atomistic simulations of (a) the apo IL3R $\alpha$ closed and open conformations (PDB ID: 4JZJ ${ }^{11}$ ), (b) wild-type IL-3:IL3R $\alpha$ binary complex (this work), (c) IL-3 K116W:IL3R $\alpha$ binary complex (this work), (d) wild-type IL-3 extracted from the binary complex crystal structure, (e) IL-3 K116W extracted from the binary complex crystal structure, (f) modelled SP2 versions of the wild-type IL-3 and IL-3 K116W binary complexes, (g) modelled apo IL3R $\alpha$ D197L and (h) modelled apo IL3R $\alpha$ C76A,C195A were carried out. Crystallographic waters, sugars and ions and so on were removed from the starting crystal structures. Missing residues or side chains were modelled in using either SYBYL-X 2.1.1 (Certara L. P., http://www.tripos.com) or Coot version $0.7^{53}$. The SP2 version of IL3R $\alpha$ (Supplementary Fig. 1c) ${ }^{24}$ was modelled using SYBYL-X 2.1.1. The D197L and C76A,C195A IL3R $\alpha$ mutations were made in silico using SYBYL-X 2.1.1. Hydrogens were added and the protein termini capped with neutral acid and amide groups. Titratable residues were left in their dominant protonation state at $\mathrm{pH}$ 7.0. All simulations were performed using the program NAMD-2.10 ${ }^{54}$ with the CHARMM $36^{55}$ force field at a constant temperature of $310 \mathrm{~K}$ and atmospheric pressure. Ions were added to balance charge and give an ionic concentration of 150 $\mathrm{mM} \mathrm{NaCl}$. The TIP3P water model ${ }^{56}$ was used in rhombic dodecahedral simulation cells with at least $12-\AA$ separation between the protein and its periodic image. For long range interactions, the Particle Mesh Ewald method was used with a switching distance of $10 \AA$ and cutoff distance of $12 \AA$. Simulations were typically performed as follows: the energy was minimised for 5000 iterations, followed by system equilibration for $1 \mathrm{~ns}$ and then production simulations of 75-200 ns were carried out. The positions of all atoms in the system were saved every $10 \mathrm{ps}$ for later analysis and visualisation using the programs $m \mathrm{mtraj}^{57}$ and $\mathrm{VMD}^{58}$.

Functional studies with IL3R $\alpha$ mutants. Human IL3R $\alpha$ cDNA was cloned into the pcDNA3.1 (Invitrogen) or pSG5 (Stratagene) expression vectors and mutations generated by $\mathrm{PCR}^{11}$. For site 1 functional studies (Fig. 3, Supplementary Table 2) and studies of the NTD interaction (Fig. 6, Table 3), we used an IL3R $\alpha$ cDNA that encodes a splice variant which lacks three nucleotides and changes N144 and R145 to K144 compared to the sequence of the sIL3R $\alpha$ used for the structural studies (Supplementary Fig. 1c). The two variants are indistinguishable in IL-3 binding and functional studies ${ }^{11}$. For the spontaneous heterodimerisation studies (Fig. 6, Supplementary Fig. 8), we used an IL3R $\alpha$ cDNA engineered to include a C-terminal Flag epitope tag (...VQKTALDYKDDDDKA-COOH) following the final threonine residue of full-length IL3R $\alpha$. Human $\beta c$ cDNA was cloned in the pSG5 vector.

Wild-type and mutant IL3R $\alpha$ was subcloned into the retroviral expression vector $\mathrm{pRufHygro}$ and the pRufHygro:IL3R $\alpha$ plasmids co-transfected into HEK293-T (ATCC CRL-3216) cells with the pEQ-Eco packaging plasmid ${ }^{59}$ using Lipofectamine 2000 (Invitrogen) to generate recombinant retrovirus. Wild-type $\beta c$ cDNA was cloned in the pRufPuro retroviral expression vector and the pRufPuro: $\beta c$ plasmid used to generate recombinant retrovirus by the same approach. Retrovirus was collected from conditioned medium at day 3 post transfection by centrifugation at 1500 r.p.m. for $5 \mathrm{~min}$ and the supernatant $0.45 \mu \mathrm{m}$ filtered. The CTLL-2 cell line (ATCC TIB-214) is maintained in RPMI media (Gibco) supplemented with antibiotics, $10 \%\left(\mathrm{v} \mathrm{v}^{-1}\right)$ foetal bovine serum, $1 \mathrm{mM}$ sodium pyruvate (Gibco), $55 \mu \mathrm{M}$ 2-mercaptoethanol (Gibco) and $400 \mathrm{U} \mathrm{ml}^{-1}$ murine IL-2. CTLL-2 cells lines were made by transduction with retrovirus. Non-tissue culture treated plates (Falcon) were coated with $32 \mu \mathrm{g} \mathrm{m}^{-1}$ RetroNectin (Takara) overnight at $4{ }^{\circ} \mathrm{C}$, blocked at room temperature with $2 \% \mathrm{w} \mathrm{v}^{-1}$ BSA for $1 \mathrm{~h}$ and then centrifuged with retrovirus at 3500 r.p.m. for $1 \mathrm{~h}$ at $37^{\circ} \mathrm{C}$. The virus coated plates were then incubated with $1 \times 10^{5} \mathrm{CTLL}-2$ cells in $1 \mathrm{ml}$ complete media overnight at $37^{\circ} \mathrm{C}$ and a second round of transduction was performed the following day using fresh retrovirus. Media was changed $24 \mathrm{~h}$ following the second round of transduction, and $48 \mathrm{~h}$ following this, antibiotic selection was added as described below. Pools of resistant cells grew out over 7-10 days and cells were sorted for receptor expression by flow cytometry. CTLL-2 cells were initially transduced to express $\beta c$, selected with puromycin (selection $2 \mu \mathrm{g} \mathrm{ml}^{-1}$, maintenance $1 \mu \mathrm{g} \mathrm{ml}^{-1}$ ) and sorted for $\beta c$ expression by flow cytometry ${ }^{60}$. Subsequently the CTLL-2: $\beta c$ cells were transduced to express the IL3R $\alpha$ variants, selected with hygromycin (selection $1000 \mathrm{\mu g} \mathrm{ml}^{-1}$, maintenance $500 \mu \mathrm{g} \mathrm{ml}^{-1}$ ) and sorted for IL3R $\alpha$ expression by flow cytometry ${ }^{60}$

The HoxA9-immortalised cell line, FDH, was generated by transduction of foetal liver cells from mice lacking $\beta \mathrm{c}$ and $\beta_{\mathrm{IL}-3}{ }^{61}$ using a pFTREtight MCS rtTAadvanced GFP lentivirus encoding a doxycycline-inducible HoxA9-Flag expression cassette ${ }^{62}$. The FDH cell line is maintained in IMDM media (Gibco) supplemented with antibiotics, $10 \%\left(\mathrm{v} \mathrm{v}^{-1}\right)$ foetal bovine serum, $100 \mathrm{ng} \mathrm{ml}^{-1}$ murine stem cell factor and $0.5 \mu \mathrm{g} \mathrm{m}^{-1}$ doxycycline hyclate (Sigma). FDH cells were transduced with $\beta c$, selected with puromycin (selection $2 \mu \mathrm{g} \mathrm{m}^{-1}$, maintenance $1 \mu \mathrm{g} \mathrm{ml}^{-1}$ ), and subsequently transduced to express the IL3R $\alpha$ variants and selected with hygromycin (selection $500 \mu \mathrm{g} \mathrm{ml}^{-1}$, maintenance $250 \mu \mathrm{g}$ $\mathrm{ml}^{-1}$ ), with sorting as described above.
Cell proliferation was assessed using CellTiter $96^{\circledast}$ AQueous (Promega) following the manufacturer's protocol. Data were analysed using GraphPad Prism to determine $\mathrm{ED}_{50}$ values.

Statistical methods. A two-tailed unpaired $t$ test was used for calculation of significance in binding and functional studies. For figures, error bars are SEM.

Cell-surface IL-3-binding assays. COS-7 cells (ATCC CRL-1651) were electroporated with pcDNA3.1:IL3R $\alpha$ plasmids encoding wild-type or mutant IL3R $\alpha$, either alone or in the presence of the pSG5H: $\beta c$ plasmid encoding human $\beta c^{60}$ Cell-surface expression of receptor subunits was confirmed by flow cytometry. Wild-type IL-3 and IL-3 K116W were radioiodinated with ${ }^{125}$ I (PerkinElmer) using Pierce pre-coated iodination tubes (Thermo Scientific). Saturation binding assays were performed on transfected COS cells, CTLL-2 cell lines, TF-1 cells or TF-1Hi cells using radioiodinated IL- $3^{60}$. Dissociation constants were calculated using the EBDA and LIGAND programs ${ }^{63}$ (KELL Radlig, Biosoft, UK). Representative data from IL-3-binding assays on TF-1 and TF-1Hi cells lines were also analysed by nonlinear regression using GraphPad Prism and yielded essentially identical dissociation constants.

Assessing the cell-surface expression of IL-3 receptor subunits. Cell-surface expression of receptor subunits was assessed by flow cytometry using $10 \mu \mathrm{g} \mathrm{m}^{-1}$ of MAb's specific for IL3R $\alpha$, including 7G3, $9 F 5^{42}$ and MAB301 (R\&D Systems cat no. MAB301), and for $\beta c, 1 \mathrm{Cl}^{64}$. Bound antibodies were stained using $5 \mu \mathrm{g} \mathrm{ml} \mathrm{m}^{-1}$ of PE conjugated goat-anti-mouse IgG (Southern Biotech cat. no. 1030-09). MAb's $7 \mathrm{G} 3$ and 9 F5 bind IL3R $\alpha$ NTD and MAB301 binds IL3R $\alpha$ D2-D3. An irrelevant mouse antibody 1B5 (anti-Giardia $\mathrm{IgG}_{1}$ ) was used as a negative control.

IL3R $\alpha$ expression and IL-3 function on TF-1 and TF-1Hi cells. The human erythroleukaemia cell line, TF-1 ${ }^{65}$ (ATCC CRL-2003) was transduced with lentiviral vectors encoding GFP alone or IL3R $\alpha$ and GFP ${ }^{26}$ to produce the TF-1EV and TF-1Hi lines, respectively. Cell-surface expression levels of IL3R $\alpha$ and $\beta c$ was measured by flow cytometry. Wild-type IL-3- and IL-3 K116W-mediated proliferation of TF-1 EV and TF-1Hi lines was assessed using CellTiter $96^{\oplus} \mathrm{AQ}$ ueous (Promega). Data were analysed using GraphPad Prism to determine $\mathrm{ED}_{50}$ values.

Constitutive association of IL3R $\boldsymbol{\alpha}$ NTD mutants with $\boldsymbol{\beta} \mathbf{c}$. HEK293-T cells were stably transduced with pRufPuro: $\beta c$ to express wild-type human $\beta c$ as described above. The HEK293-T- $\beta$ c cells were transfected with $2-10 \mu \mathrm{g}$ of pcDNA3.1:IL3R $\alpha$ Flag plasmids encoding wild-type or mutant IL3R $\alpha$ using Lipofectamine 2000 (Invitrogen). Cells were collected after 2 days and stimulated with $1.5 \mu \mathrm{g} \mathrm{ml}^{-1} \mathrm{IL}-3$ for 45 min on ice prior to lysis in NP-40 lysis buffer $\left(1 \% \mathrm{NP}-40\left(\mathrm{v} \mathrm{v}^{-1}\right), 150 \mathrm{mM}\right.$ $\mathrm{NaCl}, 50 \mathrm{mM}$ Tris- $\mathrm{HCl} \mathrm{pH} 8.0$ and $10 \%\left(\mathrm{v} \mathrm{v}^{-1}\right)$ glycerol) for $20 \mathrm{~min}$ on ice. The $\beta c$ complexes were immunoprecipitated from clarified lysate using 1C1-conjugated Sepharose beads, washed three times with lysis buffer, fractionated by SDS-PAGE and immunoblotted with antibodies for IL3R $\alpha$-Flag $\left(1 \mu \mathrm{g} \mathrm{ml}^{-1}\right.$ anti-Flag M2:HRP, Sigma cat no. A8592), $\beta \mathrm{c}\left(1.8 \mu \mathrm{g} \mathrm{ml}^{-1} \mathrm{BION}-1^{43}\right)$ and $0.1 \mu \mathrm{g} \mathrm{ml}^{-1}$ goat-anti-mouse: HRP (Pierce cat. no. 31437) or phosphorylated tyrosine residues $\left(0.25 \mathrm{\mu g} \mathrm{ml}^{-1}\right.$ antiphosphotyrosine:HRP, BD Transduction Laboratories cat. no. 610012). (Uncropped scan of immunoblot Supplementary Fig. 9). Cell-surface expression of receptor subunits was confirmed by flow cytometry of collected cells as described above.

Data availability. Atomic coordinates and structure factors of the wild-type IL-3 and IL-3 K116W binary receptor complexes have been deposited in the Protein Data Bank under the ID codes 5UV8 and 5UWC, respectively. All other data are available from the corresponding authors upon reasonable request.

Received: 4 July 2017 Accepted: 15 December 2017

Published online: 26 January 2018

\section{References}

1. Broughton, S. E. et al. The GM-CSF/IL-3/IL-5 cytokine receptor family: from ligand recognition to initiation of signaling. Immunol. Rev. 250, 277-302 (2012).

2. Hercus, T. R. et al. Signalling by the betac family of cytokines. Cytokine Growth Factor Rev. 24, 189-201 (2013).

3. Dentelli, P. et al. IL-3 is a novel target to interfere with tumor vasculature. Oncogene 30, 4930-4940 (2011)

4. Li, M. et al. Adaptive evolution of interleukin-3 (IL3), a gene associated with brain volume variation in general human populations. Hum. Genet. 135, 377-392 (2016)

5. Luo, X. J. et al. The interleukin 3 gene (IL3) contributes to human brain volume variation by regulating proliferation and survival of neural progenitors. PLoS ONE 7, e50375 (2012). 
6. Testa, U. et al. Elevated expression of IL-3Ralpha in acute myelogenous leukemia is associated with enhanced blast proliferation, increased cellularity, and poor prognosis. Blood 100, 2980-2988 (2002).

7. Jordan, C. T. et al. The interleukin-3 receptor alpha chain is a unique marker for human acute myelogenous leukemia stem cells. Leukemia 14, 1777-1784 (2000).

8. Jin, L. et al. Monoclonal antibody-mediated targeting of CD123, IL-3 receptor alpha chain, eliminates human acute myeloid leukemic stem cells. Cell Stem Cell 5, 31-42 (2009).

9. Vergez, F. et al. High levels of CD34+CD38low/-CD123+ blasts are predictive of an adverse outcome in acute myeloid leukemia: a Groupe Ouest-Est des Leucemies Aigues et Maladies du Sang (GOELAMS) study. Haematologica 96, 1792-1798 (2011).

10. Nievergall, E. et al. Monoclonal antibody targeting of IL-3 receptor alpha with CSL362 effectively depletes CML progenitor and stem cells. Blood 123, 1218-1228 (2014).

11. Broughton, S. E. et al. Dual mechanism of interleukin-3 receptor blockade by an anti-cancer antibody. Cell Rep. 8, 410-419 (2014).

12. Klein, B. K. et al. Receptor binding kinetics of human IL-3 variants with altered proliferative activity. Biochem. Biophys. Res. Commun. 288, 1244-1249 (2001).

13. Thomas, J. W. et al. Potent interleukin 3 receptor agonist with selectively enhanced hematopoietic activity relative to recombinant human interleukin 3. Proc. Natl Acad. Sci. USA 92, 3779-3783 (1995).

14. Hansen, G. et al. The structure of the GM-CSF receptor complex reveals a distinct mode of cytokine receptor activation. Cell 134, 496-507 (2008).

15. Lopez, A. F. et al. A human interleukin 3 analog with increased biological and binding activities. Proc. Natl Acad. Sci. USA 89, 11842-11846 (1992).

16. Klein, B. K. et al. The receptor binding site of human interleukin-3 defined by mutagenesis and molecular modeling. J. Biol. Chem. 272, 22630-22641 (1997).

17. Klein, B. K. et al. Use of combinatorial mutagenesis to select for multiply substituted human interleukin-3 variants with improved pharmacologic properties. Exp. Hematol. 27, 1746-1756 (1999).

18. Levin, A. M. et al. Exploiting a natural conformational switch to engineer an interleukin-2 'superkine'. Nature 484, 529-533 (2012).

19. Broughton, S. E. et al. Conformational changes in the GM-CSF receptor suggest a molecular mechanism for affinity conversion and receptor signaling. Structure 24, 1271-1281 (2016).

20. Patino, E. et al. Structure analysis of the IL-5 ligand-receptor complex reveals a wrench-like architecture for IL-5Ralpha. Structure 19, 1864-1875 (2011).

21. Kusano, S. et al. Structural basis of interleukin-5 dimer recognition by its alpha receptor. Protein Sci. 21, 850-864 (2012).

22. LaPorte, S. L. et al. Molecular and structural basis of cytokine receptor pleiotropy in the interleukin-4/13 system. Cell 132, 259-272 (2008).

23. Lupardus, P. J., Birnbaum, M. E. \& Garcia, K. C. Molecular basis for shared cytokine recognition revealed in the structure of an unusually high affinity complex between IL-13 and IL-13Ralpha2. Structure 18, 332-342 (2010).

24. Chen, J. et al. A new isoform of interleukin-3 receptor \{alpha\} with novel differentiation activity and high affinity binding mode. J. Biol. Chem. 284, 5763-5773 (2009).

25. de Vos, A. M., Ultsch, M. \& Kossiakoff, A. A. Human growth hormone and extracellular domain of its receptor: crystal structure of the complex. Science 255, 306-312 (1992).

26. Wittwer, N. L. et al. High CD123 levels enhance proliferation in response to IL-3, but reduce chemotaxis by downregulating CXCR4 expression. Blood Adv. 1, 1067-1079 (2017).

27. Thomas, C. et al. Structural linkage between ligand discrimination and receptor activation by type I interferons. Cell 146, 621-632 (2011).

28. $\mathrm{Li}, \mathrm{H}$. et al. Dynamic modulation of binding affinity as a mechanism for regulating interferon signaling. J. Mol. Biol. 429, 2571-2589 (2017)

29. Brown, R. J. et al. Model for growth hormone receptor activation based on subunit rotation within a receptor dimer. Nat. Struct. Mol. Biol. 12, 814-821 (2005).

30. Seubert, N. et al. Active and inactive orientations of the transmembrane and cytosolic domains of the erythropoietin receptor dimer. Mol. Cell. 12, 1239-1250 (2003).

31. Al-Hussaini, M. et al. Targeting CD123 in acute myeloid leukemia using a Tcell-directed dual-affinity retargeting platform. Blood 127, 122-131 (2016).

32. Busfield, S. J. et al. Targeting of acute myeloid leukemia in vitro and in vivo with an anti-CD123 mAb engineered for optimal ADCC. Leukemia $\mathbf{2 8}$ 2213-2221 (2014).

33. Tettamanti, S. et al. Targeting of acute myeloid leukaemia by cytokine-induced killer cells redirected with a novel CD123-specific chimeric antigen receptor. Br. J. Haematol. 161, 389-401 (2013).

34. Angelot-Delettre, F. et al. In vivo and in vitro sensitivity of blastic plasmacytoid dendritic cell neoplasm to SL-401, an interleukin-3 receptor targeted biologic agent. Haematologica 100, 223-230 (2015).
35. Frankel, A., Liu, J. S., Rizzieri, D. \& Hogge, D. Phase I clinical study of diphtheria toxin-interleukin 3 fusion protein in patients with acute myeloid leukemia and myelodysplasia. Leuk. Lymphoma 49, 543-553 (2008).

36. Frankel, A. E. et al. Activity of SL-401, a targeted therapy directed to interleukin-3 receptor, in blastic plasmacytoid dendritic cell neoplasm patients. Blood 124, 385-392 (2014).

37. Frolova, O. et al. SL-401 and SL-501, targeted therapeutics directed at the interleukin-3 receptor, inhibit the growth of leukaemic cells and stem cells in advanced phase chronic myeloid leukaemia. Br. J. Haematol. 166, 862-874 (2014).

38. Liu, T. F. et al. Diphtheria toxin fused to variant interleukin-3 provides enhanced binding to the interleukin-3 receptor and more potent leukemia cell cytotoxicity. Exp. Hematol. 32, 277-281 (2004).

39. Testa, U. et al. Diphtheria toxin fused to variant human interleukin-3 induces cytotoxicity of blasts from patients with acute myeloid leukemia according to the level of interleukin-3 receptor expression. Blood 106, 2527-2529 (2005).

40. Hogge, D. E. et al. Variant diphtheria toxin-interleukin-3 fusion proteins with increased receptor affinity have enhanced cytotoxicity against acute myeloid leukemia progenitors. Clin. Cancer Res. 12, 1284-1291 (2006)

41. Oon, S. et al. A cytotoxic anti-IL-3Ralpha antibody targets key cells and cytokines implicated in systemic lupus erythematosus. JCI Insight 1, e86131 (2016).

42. Sun, Q. et al. Monoclonal antibody 7G3 recognizes the N-terminal domain of the human interleukin-3 (IL-3) receptor alpha-chain and functions as a specific IL-3 receptor antagonist. Blood 87, 83-92 (1996).

43. Sun, Q. et al. Simultaneous antagonism of interleukin-5, granulocytemacrophage colony-stimulating factor, and interleukin-3 stimulation of human eosinophils by targetting the common cytokine binding site of their receptors. Blood 94, 1943-1951 (1999).

44. Broughton, S. E. et al. Crystallization and preliminary X-ray diffraction analysis of the interleukin-3 alpha receptor bound to the Fab fragment of antibody CSL362. Acta Crystallogr. F Struct. Biol. Commun. 70, 358-361 (2014).

45. Hercus, T. R. et al. High yield production of a soluble human interleukin-3 variant from E. coli with wild-type bioactivity and improved radiolabeling properties. PLoS ONE 8, e74376 (2013).

46. Newman, J. et al. Towards rationalization of crystallization screening for small- to medium-sized academic laboratories: the PACT/JCSG+ strategy. Acta Crystallogr. D Biol. Crystallogr. 61, 1426-1431 (2005).

47. McPhillips, T. M. et al. Blu-ice and the distributed control system: software for data acquisition and instrument control at macromolecular crystallography beamlines. J. Synchrotron. Radiat. 9, 401-406 (2002).

48. Otwinowski, Z. \& Minor, W. Processing of X-ray diffraction data collected in oscillation mode. Methods Enzymol. 276, 307-326 (1997).

49. McCoy, A. J. Solving structures of protein complexes by molecular replacement with Phaser. Acta Crystallogr. D Biol. Crystallogr. 63, 32-41 (2007).

50. Emsley, P. \& Cowtan, K. Coot: model-building tools for molecular graphics. Acta Crystallogr. D Biol. Crystallogr. 60, 2126-2132 (2004).

51. Murshudov, G. N. et al. REFMAC5 for the refinement of macromolecular crystal structures. Acta Crystallogr. D Biol. Crystallogr. 67, 355-367 (2011)

52. Laskowski, R. A. Structural quality assurance. Methods Biochem. Anal. 44, 273-303 (2003).

53. Emsley, P., Lohkamp, B., Scott, W. G. \& Cowtan, K. Features and development of Coot. Acta Crystallogr. D Biol. Crystallogr. 66, 486-501 (2010).

54. Phillips, J. C. et al. Scalable molecular dynamics with NAMD. J. Comput. Chem. 26, 1781-1802 (2005).

55. Best, R. B. et al. Optimization of the additive CHARMM all-atom protein force field targeting improved sampling of the backbone phi, psi and sidechain chi(1) and chi(2) dihedral angles. J. Chem. Theory Comput. 8, 3257-3273 (2012).

56. Jorgensen, W. L. et al. Comparison of simple potential functions for simulating liquid water. J. Chem. Phys. 79, 926 (1983).

57. McGibbon, R. T. et al. MDTraj: a modern open library for the analysis of molecular dynamics trajectories. Biophys. J. 109, 1528-1532 (2015).

58. Humphrey, W., Dalke, A. \& Schulten, K. VMD: visual molecular dynamics. J. Mol. Graph. 14, 33-38 (1996). 27-8.

59. Persons, D. A. et al. An improved method for generating retroviral producer clones for vectors lacking a selectable marker gene. Blood Cells Mol. Dis. 24, 167-182 (1998).

60. Woodcock, J. M., Bagley, C. J., Zacharakis, B. \& Lopez, A. F. A single tyrosine residue in the membrane-proximal domain of the granulocyte-macrophage colony-stimulating factor, interleukin (IL)-3, and IL-5 receptor common betachain is necessary and sufficient for high affinity binding and signaling by all three ligands. J. Biol. Chem. 271, 25999-26006 (1996).

61. Scott, C. L. et al. Reassessment of interactions between hematopoietic receptors using common beta-chain and interleukin-3-specific receptor betachain-null cells: no evidence of functional interactions with receptors for 
erythropoietin, granulocyte colony-stimulating factor, or stem cell factor. Blood 96, 1588-1590 (2000).

62. Brumatti, G. et al. HoxA9 regulated Bcl-2 expression mediates survival of myeloid progenitors and the severity of HoxA9-dependent leukemia. Oncotarget 4, 1933-1947 (2013).

63. Munson, P. J. \& Rodbard, D. Ligand: a versatile computerized approach for characterization of ligand-binding systems. Anal. Biochem. 107, 220-239 (1980).

64. Stomski, F. C. et al. Human interleukin-3 (IL-3) induces disulfide-linked IL-3 receptor alpha- and beta-chain heterodimerization, which is required for receptor activation but not high-affinity binding. Mol. Cell. Biol. 16, 3035-3046 (1996).

65. Kitamura, T. et al. Establishment and characterization of a unique human cell line that proliferates dependently on GM-CSF, IL-3, or erythropoietin. J. Cell. Physiol. 140, 323-334 (1989).

66. Woodcock, J. M. et al. Three residues in the common beta chain of the human GM-CSF, IL-3 and IL-5 receptors are essential for GM-CSF and IL-5 but not IL-3 high affinity binding and interact with Glu21 of GM-CSF. EMBO J. 13, 5176-5185 (1994).

\section{Acknowledgements}

This research was partly undertaken on the MX2 beamline at the Australian Synchrotron, Victoria, Australia and we thank the beamline staff for their assistance. We thank Anna Sapa for technical assistance, Denis Tvorogov for the HEK293-T cells expressing $\beta c$, Hayley Ramshaw for the TF-1Hi cell line, Paul Ekert (Murdoch Children's Research Institute) for the HoxA9 expression construct, Tony Cambareri for the 1B5 hybridoma and Joanna Woodcock for helpful discussions. We acknowledge the use of the CSIRO Collaborative Crystallisation Centre (C3), Melbourne, Australia for our initial crystallisation studies, and the SA Pathology Detmold Family Cytometry Centre for use of flow cytometry facilities. This research was supported by a Victorian Life Sciences Computation Initiative (VLSCI) grant number RA0002 on its Peak Computing Facility at the University of Melbourne, an initiative of the Victorian Government, Australia. This work was supported by grants from the National Health and Medical Research Council of Australia (NHMRC) to T.R.H., U.D., M.W.P. and A.F.L., Cure Cancer Australia to S.E.B., Cancer Council SA Beat Cancer Fund to T.P.H. and from the Australian Cancer Research Foundation to M.W.P. Funding from the Victorian Government Operational Infrastructure Support Scheme to St Vincent's Institute is acknowledged. S.E.B is a Postdoctoral Fellow supported by the Leukaemia Foundation. T.P.H. is an NHMRC Practitioner Fellow and M.W.P. is an NHMRC Research Fellow.

\section{Author contributions}

S.E.B. performed the crystallographic studies and the structural analyses. T.R.H. prepared proteins and complexes for structural and functional studies. T.R.H., W.L.K., M.D., C.J. M. M.P.H., N.J.W. and E.F.B. did binding and functional studies. T.L.N. did molecular modelling. T.L.N., U.D., C.J.M. and K.S.C.T.S. assisted with structural analyses. T.R.H., M.W.P. and A.F.L. designed experiments. M.T.D. and C.S. performed the MD simulations and analyses. K.S.C.T.S., S.E.B., T.R.H., C.S. and T.L.N. prepared figures. S.E.B., T.R H., T.P.H., T.L.N., M.W.P., and A.F.L. wrote the paper. All authors discussed the results and commented on the manuscript. M.W.P. and A.F.L. supervised the research.

\section{Additional information}

Supplementary Information accompanies this paper at https://doi.org/10.1038/s41467017-02633-7.

Competing interests: M.P.H. and N.J.W. are employees of CSL Limited and M.T.D. and C.S. are employees of IBM Research Australia. The remaining authors declare no competing financial interests.

Reprints and permission information is available online at http://npg.nature.com/ reprintsandpermissions/

Publisher's note: Springer Nature remains neutral with regard to jurisdictional claims in published maps and institutional affiliations.

(c) (i) Open Access This article is licensed under a Creative Commons Attribution 4.0 International License, which permits use, sharing, adaptation, distribution and reproduction in any medium or format, as long as you give appropriate credit to the original author(s) and the source, provide a link to the Creative Commons license, and indicate if changes were made. The images or other third party material in this article are included in the article's Creative Commons license, unless indicated otherwise in a credit line to the material. If material is not included in the article's Creative Commons license and your intended use is not permitted by statutory regulation or exceeds the permitted use, you will need to obtain permission directly from the copyright holder. To view a copy of this license, visit http://creativecommons.org/ licenses/by/4.0/.

(c) The Author(s) 2018 